\title{
Deleting in vivo $\beta$-catenin degradation domain in mouse hepatocytes drives hepatocellular carcinoma or hepatoblastoma-like tumors
}

Robin Loesch ${ }^{1-2}$, Stefano Caruso ${ }^{1}$, Valérie Paradis ${ }^{3}$, Cecile Godard ${ }^{1-2}$, Angélique Gougelet ${ }^{1-2}$, Simon Picard ${ }^{1-2}$, Christine Perret ${ }^{4}$, Makoto Mark Taketo ${ }^{5}$, Jessica Zucman-Rossi ${ }^{1}$, Sabine Colnot $^{1-2^{*}}$

${ }^{1}$ INSERM, Sorbonne Université, Université de Paris, Centre de Recherche des Cordeliers (CRC), F-75006; ${ }^{2}$ Equipe labellisée Ligue Nationale Contre le Cancer, France ; ${ }^{3}$ INSERM, Hôpital Beaujon, Clichy, France ; ${ }^{4}$ INSERM, CNRS, Institut COCHIN, F-75014 ; ${ }^{5}$ Colon Cancer Project, Kyoto University Hospital-iACT, Kyoto, Japan.

*Corresponding author : Sabine COLNOT. Centre de recherche des Cordeliers, INSERM UMRS 1138, 15 Rue de I'Ecole de Médecine, 75006 PARIS, France. sabine.colnot@inserm.fr ; Phone : +33144274005.

\section{Keywords}

Hepatocellular Carcinoma, Hepatoblastoma, beta-catenin, Hippo pathway, GeneticallyENgineered Mice Models, Cre-loxP, CRISPR-Cas9

Electronic word count: 5782

Number of Figures \& Tables: 7 figures

\section{No conflict of interest}

\section{Authorship contributions:}

$\mathrm{RL}$ and SCo conceived the experiments and study design. Experiments were carried out by RL. SCa analyzed transcriptome data collected by JZR. VP is the pathologist who histologically characterized mouse HCCs. CG and AG performed mouse breeding, tumor follow-up, RNA isolation and immunohistochemistry studies in Cre-loxP-generated mice. SP performed immunohistochemistries in AAV-CRISPR-Cas9 mouse models. CG performed liver biopsies. MT provided $\beta \mathrm{cat}^{\text {ex3-flox }}$ mouse model at the request of $\mathrm{CP}$, who initiated the study with $\mathrm{SCO}$. Sco supervised the study. RL and SCo wrote the manuscript with input from authors.

\section{Fundings:}

The project was supported by the Ligue Nationale Contre le Cancer (Labelisation), by the Institut National pour la Santé et la Recherche Médicale (INSERM), and by the Institut National du Cancer (INCa Emergence 2017). RL was supported by a fellowship from Université de Paris and from the Fondation pour la Recherche Médicale. 


\section{Abstract}

Background and aims: One-third of hepatocellular carcinomas (HCCS) have mutations that activate the $\beta$-catenin pathway with mostly CTNNB1 mutations. Mouse models using Adenomatous polyposis coli (Apc) loss-of-functions (LOF) are widely used to mimic $\beta$-catenindependent tumorigenesis. Considering the low prevalence of $A P C$ mutations in human HCCS we aimed to generate hepatic tumors through CTNNB1 exon 3 deletion $\left(\beta c a t^{\Delta \mathrm{ex} 3}\right)$ and to compare them to hepatic tumors with Apc LOF engineered through a frameshift in exon 15 $\left(A p c^{f s-e x 15}\right)$.

Methods: We used hepatic-specific and inducible Cre-lox mouse models as well as a hepaticspecific in vivo CRISPR/Cas9 approach using AAV vectors, to generate $A p c^{f s-e x 15}$ and $\beta$ cat ${ }^{\Delta e \times 3}$ hepatic tumors harboring activation of the $\beta$-catenin pathway. Tumors generated by the Crelox models were analyzed phenotypically using immunohistochemistry and were selected for transcriptomic analysis using RNA-sequencing. Mouse RNAseq data were compared to human RNAseq data (normal tissues (8), HCCs (48) and hepatoblastomas (9)) in an integrative analysis. Tumors generated via CRISPR were analyzed using DNA sequencing and immunohistochemistry.

Results: Mice with $\beta$ cat $^{\Delta \mathrm{ex} 3}$ alteration in hepatocytes developed liver tumors. Generated tumors were indistinguishable from those arising in $A p c^{f s-e x 15}$ mice. Both $A p c^{f s-e x 15}$ and $\beta c a t^{\Delta e x 3}$ mouse models induced two phenotypically distinct tumors (differentiated or undifferentiated). Integrative analysis of human and mouse tumors showed that mouse differentiated tumors are close to human well differentiated CTNNB1-mutated tumors, while undifferentiated ones are closer to human mesenchymal hepatoblastomas, and are activated for YAP signaling.

Conclusion: $A p c^{f s-e x 15}$ and $\beta$ cat $^{\Delta \mathrm{ex} 3}$ mouse models similarly induce tumors transcriptionally close to either well differentiated $\beta$-Catenin activated human HCCs or mesenchymal hepatoblastomas. 


\section{Introduction}

Hepatocellular carcinoma ( $\mathrm{HCC}$ ), the most frequent liver primary cancer, is the fourth highest cause of cancer-related death ${ }^{1}$. It mainly arises on a diseased liver, associated with hepatitis $B(\mathrm{HBV})$ and $\mathrm{C}(\mathrm{HCV})$ viral infections, alcohol abuse or metabolic syndrome. HCC development relies on a multistep process involving genetic alterations. Prevailing mutational hotspots highlight telomerase activation, TP53 or $\beta$-catenin signaling, and chromatin remodeling process, as being the core pathways supporting HCC pathogenesis. The oncogenic property of dysregulated Wnt/ $\beta$-Catenin pathway in HCC was first suggested after the discovery of activating somatic point mutations in CTNNB1 gene encoding $\beta$-Catenin both in human and mouse $\mathrm{HCC}^{2}$. Nowadays, CTNNB1 mutations in human $\mathrm{HCC}$ are well described and occur mainly in its exon 3 , disrupting the degradation domain of $\beta$-catenin. Mutations lead to $\beta$-catenin stabilization, to its translocation in the nucleus where it participates to a transcriptional complex with the LEF/TCF nuclear factors, regulating gene expression. Some target genes of nuclear hepatic $\beta$-catenin such as GLUL encoding Glutamine Synthetase (GS) and $A X I N 2$, are markers of CTNNB1-mutated human $\mathrm{HCCS}^{3,4}$. Even though the majority of the mutations that activate the $\beta$-Catenin pathway are found in CTNNB1 itself ( $37 \%$ of HCCs), mutations in Adenomatous Polyposis Coli (APC) and AXIN1, which are involved in $\beta$-catenin degradation complex, are also found in $1-2 \%$ and $11 \%$ of HCCs respectively ${ }^{5}$. A recent study showed that AXIN1 deficiency in human and mouse induces HCC without $\beta$-Catenin activation $^{6}$. Conversely, we previously described the inducible and liver-specific loss of function of $A p c$, obtained through a Cre-loxP mediated excision of its exon 14, generating a frameshift in exon 15, thereafter called the $A p c^{\text {fs-ex } 15}$ mouse model: $A p c$ loss in single hepatocytes led to $\beta$-catenin signaling and liver tumorigenesis ${ }^{7}$. Since APC mutations are found in only $1 \%$ of human HCCs, we could not exclude that $\beta$-catenin dependent tumorigenesis in the $A p c^{f s-e x 15}$ mouse does not phenocopy that linked to CTNNB1 gene alterations. It could be due to multiple alternative functions exerted by APC, including its role in chromosomal instability found in colorectal cancers ${ }^{8}$.

About $10 \%$ of CTNNB1 mutations found in human HCCs or hepatocellular adenomas (HCA) are large deletions in exon 3 leading to in-frame deletion of sequences encoding the residues whose phosphorylation is needed for $\beta$-Catenin degradation (serine 33-37-45 and threonine 41$)^{4}$. Hepatoblastoma is a pediatric liver primary cancer in which CTNNB1 is also a 
key driver gene through in-frame mutation (50-90\%) mainly consisting in deletions surrounding the exon $3^{9}$. In 2002, Harada et al generated a mouse model generating a CreloxP-mediated hepatic loss of the exon 3 of $C \operatorname{tnnb1}\left(\beta c a t^{\Delta e x 3}\right)$, in which no liver tumorigenesis occurred 6 months after adenovirus-CRE (AdCre) injection ${ }^{10}$. Here we used an alternate tamoxifen-inducible approach for Cre recombination to test liver tumorigenesis in $\beta c a t^{\Delta e x 3}$ mice, similar to a protocol efficient for inducing liver tumorigenesis in $A p c^{f s-e x 15}$ mice $^{3,11-13}$. We also edited Apc or Ctnnb1 in hepatocytes by performing in vivo an AAV-mediated delivery of CRISPR/Cas9 machinery ${ }^{14}$.

We showed that both Cre-loxP- and CRISPR-Cas9-mediated $\beta c a t^{\Delta e x 3}$ alteration in hepatocytes induce $\beta$-catenin-dependent liver tumorigenesis similarly to the $A p c^{f s-e x 15}$ mouse model. Regardless of the Wnt partner targeted and of the gene editing strategy, the mice generated two phenotypically distinct types of tumors that we defined as well-differentiated (DIFF) or poorly-differentiated (UNDIFF) tumors. Integrative analysis of RNAseq data from mouse tumors with human HCCs or hepatoblastomas revealed that DIFF tumors in mouse are close to well differentiated CTNNB1-mutated human HCCs, while UNDIFF mouse tumors are close to human mesenchymal hepatoblastomas. 


\section{Material and methods}

In vivo CRISPR design and editing analysis. All the guides used were designed using CRISPR RGEN online tool (Cas-Designer http://www.rgenome.net/cas-designer/ ). Twenty-one nucleotides long guides were selected according to their GC content (20\% to $80 \%$ ), low potential off-target sites and position in previously tested PCR for easy and efficient indels analysis via T7 nuclease assay or SANGER sequencing followed by online analysis by TIDE (Tracking of Indels by DEcomposition; https://tide.deskgen.com/). Guides were cloned in single AAV-Cas9 plasmids containing both sgRNA and Cas9 from Staphylococcus aureus, driven by either a CMV promoter (pX601, addgene \#61591, used for in vitro studies), or a TBG promoter (pX602, addgene \#61593, Figure 2A) ${ }^{14}$. Plasmids were then transfected independently into mouse hepatoma Hepa1-6 cells for Apc and Rosa26 guides, or mouse AML12 cell line for the $\beta c^{-a t}{ }^{\Delta e x 3}$ strategy. For each locus, three guides were tested in vitro to select the most efficient ones (Table S1). Two days after transfection, cell DNA was extracted and amplified by PCR for the targeted loci.

Cell culture and transfection. Hepa1-6 cells were maintained in DMEM medium supplemented with $10 \%$ fetal calf serum and $1 \%$ penicillin-streptomycin. AML12 cells were maintained in DMEM/F12 medium (1:1) supplemented with $10 \%$ fetal calf serum and $1 \%$ penicillin-streptomycin. Hepa1-6 and AML12 cells were transfected in six well plates using $10 \mu$ l lipofectamine 2000 (Life Technologies). Due to a mutation deleting a part of Ctnnb1 exon 3 in Hepa1-6 (Figure S5C), AML12 cells were used to test the guide RNAs targeting intron 2 and 3.

Editing analysis. The PCR product of the targeted loci were sequenced by Sanger for assessment of total DNA insertions and deletions (indels), using TIDE or analyzed by a T7 nuclease assay (Surveyor ${ }^{\circledR}$ Mutation Detection Kit - IDT 706020). Surveyor assays were performed for $A p c$ and Rosa26 initial experiments only, as it appeared more quantitative and reproducible to use Tide. For Sanger sequencing, the DNA was extracted on gel after PCR. Guides and PCR primers are in Table S1. To measure in vivo the efficiency of Ctnnb1 exon3 editing, specific PCRs were performed from $\beta$ cat $^{\Delta \mathrm{ex} 3}$ livers and tumors: Taqman assays (Thermo Fisher Scientific) amplified either WT Ctnnb1 (Mm00483025), or WT and exon 3deleted Ctnnb1 (Mm01350394). 
Production of AAV-CRISPR/Cas9 viral particles. pX602 plasmids were amplified in Stbl3 bacterial strain then purified using Qiagen Plasmid Maxi Kit (ID:12162) and sent to the Center of viral vector production at the Health Research Institute in Nantes for recombinant AAV8 production (CPV INSERM UMR1089 Université de Nantes).

Animal injection and processing. All animal procedures were approved by the ethical committee of Université de Paris according to the French government regulation (APAFIS \#16420). The mice were maintained at the animal facility with standard diet and housing. Retro-orbital injections, ultrasonography and liver biopsies were done under general anesthesia with isoflurane inhalation. The $A p c^{\text {fs-ex } 15}$ mouse model was generated from compound $A p c^{\text {flox/flox } / T T R-C r e ~}{ }^{\text {Tam }}$ injected with $0.75 \mathrm{mg}$ Tamoxifen via intraperitoneal injection after dilution in oil ${ }^{12,13}$. $\beta$ cat $^{\text {exon3-flox }}$ mice were intercrossed with TTR-Cre ${ }^{\text {Tam }}$ mice to generate a tamoxifen inducible and hepatocyte-specific model for Ctnnb1 exon 3 deletion $\left(\beta c a t^{\Delta \mathrm{ex} 3}\right)^{10 \text {, }}$ 15. Two month-old $\beta$ cat ${ }^{\text {exon3-flox} / T T R-C r e ~}{ }^{\text {Tam }}$ male mice were also injected with Tamoxifen. Alternatively, they were given a tamoxifen diet (M-Z, low phytoestrogen $+400 \mathrm{mg} / \mathrm{kg}$ TAM citrate, SSNIFF, Soest, Germany) for two days after an overnight fasting. For the CRISPR-Cas9 strategy, retro-orbital injections of AAVs diluted in $200 \mu$ l of physiological serum were done in 2-month-old C57BI6/N male mice. Liver biopsies of the ligated extremity of the left median lobe $\left(0.1 \mathrm{~cm}^{3}\right)$ were performed after minimal laparotomy. Excepted for the Cre-loxP $\beta$ cat ${ }^{\Delta e x 3}$ mice, $A p c^{f s-e x 15}$ and $\beta c a t^{\Delta e x 3}$ mice were followed by ultrasonography every month until tumor detection, thereafter ultrasound imaging was continued every 2 weeks, as described ${ }^{12}$. Mice were sacrificed when tumor mass exceeded $2 \mathrm{~cm}^{3}$ par mouse. The lethality rates indicate either a sudden death, or a sacrifice when ethical endpoints were reached.

Immunohistochemistry (IHC) and staining. Hematoxylin-Eosin stainings and IHC were done on Formalin-fixed paraffin-embedded (FFPE) liver tissue sections as previously described ${ }^{2}$. The antibodies used are in Table S2.

RNA and DNA extraction. Total RNAs were extracted from tumor or non-tumor tissue with Trizol reagent (ThermoFisher Scientific - 15596018) as previously described ${ }^{12}$. DNA extractions were performed using classical phenol-chloroform protocol (Invitrogen Ultrapure Phenol:Chloroform:Isoamyl Alcohol (25:24:1, v/v)). 
Mouse RNA sequencing. Libraries were prepared by Integragen SA with NEBNext Ultra II Directional RNA Library Prep Kit for Illumina protocol according supplier recommendations. Briefly the key stages of this protocol are successively, the purification of PolyA containing mRNA molecules using poly-T oligo attached magnetic beads from $1 \mu g$ total RNA (with the Magnetic mRNA Isolation Kit from NEB), a fragmentation using divalent cations under elevated temperature to obtain approximately 300bp pieces, double strand cDNA synthesis and finally Illumina adapters ligation and CDNA library amplification by PCR for sequencing. Sequencing was then carried out on paired-end 75 b of Illumina HiSeq4000. Base calling was performed by using the Real-Time Analysis software sequence pipeline (2.7.7) with default parameters. Quality of reads was assessed for each sample using FastQC (V.0.11.4; http://www.bioinformatics.babraham.ac.uk/projects/fastqc/). Mouse RNAseq analysis was performed using Galileo online software provided by Integragen. Data have been deposited in the European Nucleotide Archive (ENA) at EMBL-EBI under accession number PRJEB44400. (https://www.ebi.ac.uk/ena/browser/view/PRJEB44400)

Human-Mouse integrative analysis. Statistical analysis and data visualization were performed using R software version 3.5.1 (R Foundation for Statistical Computing, Vienna, Austria. https://www.R-project.org) and Bioconductor version 3.4. A total of 80 samples from 2 different RNA-sequencing datasets was used for the comparative transcriptomic analysis, including 65 human and 15 mouse samples, respectively. The two datasets were initially processed separately. For each dataset, FPKM scores (number of fragments per kilobase of exon model and millions of mapped reads) were calculated by normalizing the count matrix for the library size and the coding length of each gene. FPKMs were, subsequently, logtransformed after adding a pseudocount of 1 . First, we filtered out most of the genes by keeping only the 1000 genes with the highest variance in both datasets and, then, only common genes that are 1:1 orthologs in human and mouse were selected $(n=324)$ using the list of mouse-human 1:1 orthologous genes from MGI (http://www.informatics.jax.org). Finally, we standardized gene expression separately to have mean 0 and standard deviation 1 per gene just before the two datasets were integrated. Unsupervised hierarchical clustering of the integrated data was performed using Pearson distance and Ward's linkage method with ComplexHeatmap package in R. Principal component analysis (PCA) using the main two components was also performed. Pairwise correlations between mouse and human samples 
bioRxiv preprint doi: https://doi.org/10.1101/2021.07.04.450836; this version posted July 5, 2021. The copyright holder for this preprint (which was not certified by peer review) is the author/funder. All rights reserved. No reuse allowed without permission.

Loesch et al., J Hepatol

were determined using the expression profiles of the 324 mouse-human 1:1 orthologous genes and the Corrplot package in R. Hierarchical clustering of the obtained correlation coefficients was done using euclidean distance and average linkage method. 


\section{Results}

\section{$\beta$ cat $^{\Delta \mathrm{ex} 3}$ CreloxP-engineered gene alteration in mouse induces liver cancer}

We first asked whether $\beta \mathrm{cat}^{\Delta \mathrm{ex3}}$ mice develop liver tumors, as $A \mathrm{pc}^{\mathrm{fs}-\mathrm{ex} 15}$ mice do. Lethality had

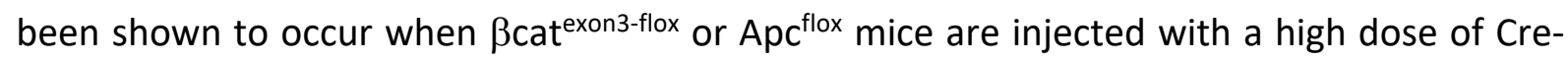
Adenovirus (AdCre), as most of the hepatocytes are $\beta$-catenin-activated ${ }^{7}, 10$. We previously showed that a 2 -fold lower dose of AdCre was sufficient to delete $A p c$, leading to $\beta$-catenin activation in single hepatocytes, and to tumorigenesis within 9 months ${ }^{7}$. A 10-fold lower dose of AdCre had been previously used for $\beta$ cat ${ }^{\text {exon3-flox }}$ mice: this dose activated $\beta$-catenin in rare hepatocytes and was not tumorigenic 6 months after injection ${ }^{10}$. We therefore decided to

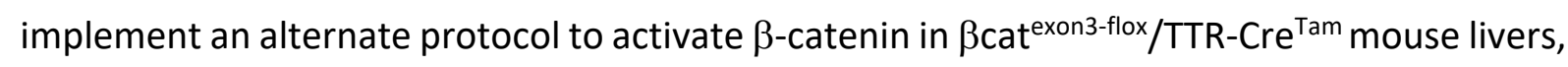
using Tamoxifen to activate Cre recombinase from TTR-Cre ${ }^{\text {Tam }}$ transgene ${ }^{15}$. We also performed a longer follow-up of tumors, until 12 months after Tamoxifen treatment.

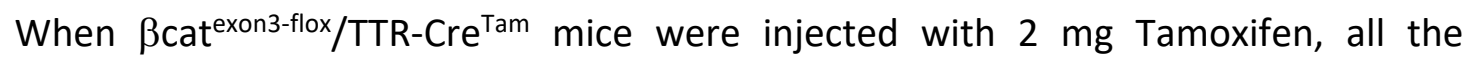
hepatocytes excepted the most periportal ones were $\beta$-catenin-activated (Figure S1). To activate $\beta$-catenin signaling in single hepatocytes and study tumorigenesis, $\beta$ cat ${ }^{\text {exon3-flox }} /$ TTR$\mathrm{Cre}^{\mathrm{Tam}}$ mice were either injected with $1 \mathrm{mg}$ or $0.5 \mathrm{mg}$ tamoxifen, or were given a tamoxifenenriched diet for 2 days (Figure 1). High lethality still occurred in mice within one month after injection with $1 \mathrm{mg}$ or $0.5 \mathrm{mg}$ tamoxifen, with the long term survival of $23 \%$ and $58 \%$ of the mice respectively. Feeding mice with tamoxifen-enriched diet for 2 days increased mice viability to $90 \%$.

Nineteen out of 31 surviving $\beta c^{\Delta t^{\Delta e x 3}}$ mice developed hepatic tumors during the 12 months following tamoxifen exposure. The status of the $\beta$-catenin signaling in tumors $(T)$ and non-tumor (NT) tissue was assessed using $\beta$-catenin and glutamine synthetase (GS) staining (Figure 1, Figure S6). Cytoplasmic and/or nuclear localization of $\beta$-catenin as well as GS expression was found in few hepatocytes in NT liver. Like our previous observations with $A p c^{f_{s-}}$ ex15 mouse model, we observed the development of two types of tumors, either welldifferentiated (DIFF) or poorly-differentiated (UNDIFF) ${ }^{7}$. Well-differentiated tumors were characterized by hepatocyte-like tumor cells that maintain the $\beta$-catenin-induced expression of GS and present slight cytosolic or nuclear $\beta$-Catenin. Undifferentiated tumors were 
composed of small cells with basophilic nuclei. They strongly express nuclear $\beta$-Catenin but lose the expression of GS which is reminiscent of a loss of differentiation (Figure 1, Figure S6). Among nine isolated tumors, 5 were classified as DIFF and 4 as UNDIFF (Figure 3A). The data clearly emphasize that a dominant stable mutant of $\beta$-catenin is sufficient to induce liver carcinogenesis in mouse.

\section{$\beta$ cat $^{\Delta \mathrm{ex} 3}$ and $A p c^{\text {fs-ex15 }}$ mouse models can be generated through CRISPR/Cas9}

In order to implement an easy-to-use model of $\beta \mathrm{cat}^{\Delta \mathrm{ex} 3} \mathrm{HCC}$, we performed CRISPR/Cas9 strategy in vivo to target the liver, using AAV8 hepatotropic vectors able to edit genes only in hepatocytes thanks to the hepatospecific TBG promoter driving the Cas9 expression (Figure $\mathbf{2 A})^{14}$. As a proof of concept that such an approach can be used to induce long term carcinogenesis, we first generated the $A p c^{\mathrm{fs}-\mathrm{ex} 15}$ model. We selected specific guide RNA (sgRNA or sg) to target the beginning of the exon 15 of $A p c(\operatorname{sgApc})$ or the Rosa26 locus ( $\operatorname{sg} R o s a$ ) as a control (Figure 2B and figure S2A). After production of rAAV8 viral particles, C57/BI6 mice were injected with $2.10^{11}$ viral genome (vg) of AAV-CRISPR-sgApc/Rosa. Two months after injection, editing efficiency on total liver was first quantified using TIDE algorithm (Figure 2C-E-F). Mean efficiency was $14.5 \pm 3.3 \%$ for $\operatorname{sgApc}$ and $35.1 \pm 3.9 \%$ for sgRosa showing a guide-dependent variability (Figure $\mathbf{2 C}$ ). Despite this variability, the mutational profile induced by each guide as well as the total editing was highly reproducible (Figure 2E-F). We performed liver biopsies two weeks after injection of AAV-CRISPR-sgApc. Single hepatocytes expressing GS were found randomly distributed in hepatic lobules, indicating focal activation of the $\beta$-catenin pathway (Figure $\mathbf{S 3} \mathbf{a}, \mathbf{b}$ ). At one month, these livers revealed a slightly higher editing efficiency (Figure S3c,d). As expected, control AAV-CRISPR-sgRosa-injected mice had livers undistinguishable from wild type mice, as GS immunolocalization was restricted to the hepatocytes surrounding the central vein, which corresponds to the physiologic area with Wnt/ßcatenin activation ${ }^{11}$ (Figure S4B).

Next, guides targeting intron 2 (sgl2) and intron 3 (sgl3) of Ctnnb1 gene were selected to engineer a $\beta$ cat $^{\Delta \mathrm{ex} 3}$ model (Figure 2B, S2B-D). Two months after injection of $2.10^{11}$ viral particles (vg) of single AAV-CRISPR-sgI2 or AAV-CRISPR-sgI3 in C57/BI6 mice, editing efficiency was higher than $40 \%$ indels (Figure $\mathbf{2 C}$ ). We then injected simultaneously $10^{11} \mathrm{vg}$ of each AAVCRISPR-sgI2 and AAV-CRISPR-sgI3. Such a dose induced $\beta$-catenin activation in a small subset of hepatocytes 2 weeks post injection to up to $40 \%$ of Ctnnb1-exon3 excision on total liver 
cells after 1 month (Figure 2D, S4A). This led to a massive activation of the $\beta$-catenin pathway in more than $90 \%$ of hepatocytes and to the death of the injected animals. Lowering the dose to $2.10^{10}$ vg per AAV circumvented mortality. Resulting CRISPR- $\beta$ cat ${ }^{\Delta \mathrm{ex} 3}$ livers showed a significant number of single hepatocytes activated for $\beta$-catenin signaling, suitable for long term tumorigenesis studies (Figure S4B).

We performed a follow-up of CRISPR-Apc $c^{\mathrm{fs}-\mathrm{ex} 15}$, CRISPR- $\beta \mathrm{cat}^{\mathrm{\Delta ex3}}$ and control CRISPRRosa26 mice using ultrasound until tumor development (Figure 2H). As expected, all CRISPRRosa26 mice remained healthy until sacrifice 16 months after injection $(n=4)$ showing no deleterious effect of the CRISPR/Cas AAV-mediated in vivo strategy. On the contrary, all CRISPR-Apc $\mathrm{fs}^{\mathrm{fs} 15}(\mathrm{n}=9)$ and CRISPR- $\beta \mathrm{cat}^{\Delta \mathrm{ex} 3}(\mathrm{n}=11)$ mice developed tumors on average $355 \pm 73$ days and $317 \pm 79$ days after injection respectively. Editing analysis on 13 CRISPR-Apc fs-ex15 tumors using Tide revealed 1 to 4 indels per tumor, inducing frameshifts, with $75 \%$ of edited DNA in average, showing that tumors emerge from edited hepatocytes (Table $\mathbf{S 3}$ and Figure 2I). Analysis of exon 3 deletion in CRISPR- $\beta c{ }^{\Delta{ }^{\Delta e x} 3}$ tumors and NT adjacent tissue showed a greater PCR amplification in tumors of the $\sim 571$ bp $\Delta$ exon3 fragment expected $(n=14)$ (Figure 21). This was confirmed by Sanger sequencing of DNA in 8 tumors, which also revealed the insertion of a DNA sequence similar to the AAV-ITR in one tumor (\#269 T3 - Figure 2G). Noteworthy, we targeted independently intron 2 or 3 to test the efficiency of the guides in vivo and no $\beta$-catenin activation was seen 2 months after injection (Figure 2C, Figure S5A). In this setting, one mouse that received a single AAV targeting intron 2 developed a $\beta$-cateninactivated tumor 8 months after injection (Figure S5B) and we found a deletion of more than $100 \mathrm{bp}$ at the beginning of exon 3. This oncogenic deletion is reminiscent of the heterozygous 45bp deletion that we identified in the murine Hepa 1-6 cell line (Figure S5C).

As described in CreLox-generated $A p c^{\mathrm{fs}-\mathrm{ex} 15}$ and $\beta c a t^{\Delta \mathrm{ex} 3}$ mouse models, differentiated and undifferentiated tumors are observed when using CRISPR, showing an intense immunostaining for $\beta$-Catenin in UNDIFF tumors, and a slightly reinforced one in DIFF tumors (Figure 2I), indicating activation of the $\beta$-Catenin pathway. Our findings demonstrate that expressing a dominant stable mutant of $\beta$-Catenin is sufficient to induce tumorigenesis with a complete penetrance, and that CRISPR technology can easily and reproducibly be used to induce and maintain long term gene editing and carcinogenesis in the liver.

$\beta$ cat $^{\Delta e \times 3}$ mice develop tumors similar to $A p c^{f s-e x 15}$ mice 
We analyzed mice with $\beta$-catenin-activated hepatic tumors, after the expression of a stable $\beta$-Catenin $\left(\beta c a t^{\Delta \mathrm{ex} 3}\right)$ or a loss-of-function of $A p c\left(A p c^{f s-e x 15}\right)$, using either CreLox or CRISPR strategy. We could not detect lung metastases in either model, either macroscopically or by the analysis of hemalun-eosine stained FFPE-sections. We similarly found that $A p c^{\text {fs-ex15 }}$ and $\beta \mathrm{cat}^{\triangle \mathrm{ex} 3}$ mice each gave rise to either DIFF or UNDIFF liver tumors (Figure 3A, Figure S6). Tumors were of similar size and were hyperproliferative (Figure $\mathbf{3 B}, \mathbf{C}$ ). Some tumor cells in UNDIFF tumors showed a positive immunostaining for the cleaved activated form of Caspase 3 , revealing a mild apoptosis rate in these tumors (Figure $\mathbf{3 C}$ ).

We sought to examine the differences between $A p c^{f s-e x 15}$ and $\beta c a t^{\Delta e x 3}$ tumors from a transcriptomic point of view by RNA-sequencing of DIFF and UNDIFF tumors from both Crelox models. Strikingly, unbiased principal component analysis, hierarchical and consensus clusterings clearly separated into three distinct groups: NT tissue, DIFF tumors and UNDIFF tumors, independently on the model (Figure 4). Accordingly, almost no genes were found differentially expressed by comparing $\beta c \mathrm{ct}^{\Delta \mathrm{ex} 3}$ and $A p c^{\mathrm{fs}-\mathrm{ex} 15}$ tumors with similar phenotype ( 2 genes over-expressed in $A p c^{f s-e x 15}$ DIFF tumors compared to $\beta$ cat $^{\Delta e x 3}$ tumors and 0 genes between $\beta c a t^{\Delta e x 3}$ and $A p c^{f s-e x 15}$ UNDIFF tumors, q-value<0.05, -1>Log2(FC)>1) (Figure S7). Our data showed that tumors induced in $\mathrm{Apc}^{\mathrm{fs}-\mathrm{ex} 15}$ model are similar to tumors induced in $\beta \mathrm{cat}^{\Delta \mathrm{ex} 3}$ model.

\section{Ctnnb1 $1^{\Delta E \times 3}$ and $A p c^{\text {fs-ex15 }}$ mouse models induce tumors transcriptionally close to human} liver primary tumors

We previously described that DIFF mouse tumors looked like $\beta$-catenin-mutated human HCCs, described as well-differentiated tumors ${ }^{7}$. We searched to which type of liver primary tumors, UNDIFF $\beta$ catenin-activated mouse tumors could be related. A Geneset Enrichment Analysis (GSEA) found a human hepatoblastoma signature in these UNDIFF mouse liver tumors (Figure 4D).

We analyzed more deeply how murine hepatic tumors could be associated to subtypes of human liver primary cancers by doing an integrative analysis (Figure S8). We combined our recently characterized human hepatoblastoma (HB) cohort ${ }^{16}$ and $\mathrm{HCC}$ datasets from our previously described molecular classification $\mathrm{G} 1$ to $\mathrm{G} 6^{17,18}$, with RNAseq data of mouse $\beta$ cat- 
HCCs. Using hierarchical clustering, we clearly showed that: (1) DIFF mouse tumors are close to human well-differentiated $\beta$-Catenin-activated G5-G6 HCCs ( $\beta$ cat-hHCC) constituting the cluster 4 ; (2) UNDIFF mouse tumors are close to human hepatoblastomas, particularly to mesenchymal hepatoblastomas (M-hHB), constituting the cluster 1 (Figure 5). A similar clustering was obtained when generating a correlation matrix between human and mouse samples (Figure S9A). As expected from their $\beta$-catenin-activated status, both UNDIFF and DIFF mouse HCCs had a $\beta$-catenin transcriptional signature, but this signature was qualitatively different. The DIFF tumors were enriched in $\beta$-catenin signaling targets found in the adult liver after Apc loss, expressing pericentral markers such as $\mathrm{GS}^{3}$ (Figure S9B). This pericentral $\beta$ catenin signature is also found in human $\beta$ cat-hHCCs ${ }^{3}$. Conversely, UNDIFF tumors were enriched in the expression of $\beta$-catenin targets found in the embryonic liver after Apc loss ${ }^{19}$ (Figure S9B): some of which are found common in M-hHB, such as NKD1, TNFRSF19, APCDD1 (Figure 5). Besides this common $\beta$-catenin progenitor signature, we found a common Epithelial-Mesenchymal Transition (EMT) signature in UNDIFF tumors and M-hHBs (Figure 6B). This included an increase in mesenchymal markers such as MMP2 and Vimentin (VIM), an increase in transcription factors involved in EMT, such as SNAIL and SLUG (SNAI2), ZEB1 and ZEB2, together with a decrease in epithelial markers such as Occludin (OCLN) and Ecadherin (CDH1) (Figure 6B). We confirmed this pattern, as we found a strong Vimentin immunostaining only in UNDIFF tumors (Figure 6C). This underlines the mesenchymal status of murine UNDIFF tumors.

To summarize, $A p c^{\mathrm{fs}-\mathrm{ex} 15}$ and $\beta \mathrm{cat}^{\Delta \mathrm{ex} 3}$ mouse tumors similarly generate two distinct types of tumors transcriptionally close to human $\beta$-catenin-activated tumors, either welldifferentiated G5-G6 HCCs, or mesenchymal HBs.

\section{YAP/TAZ signaling is activated in undifferentiated $\beta$-catenin-activated mouse liver} tumors

We finally looked for a pathway directing the phenotypic features of UNDIFF mouse tumors, and connected to $\beta$-catenin pathway in Hepatoblastoma. The YAP/TAZ pathway was a tempting hypothesis, as it is activated in hepatoblastomas and linked to $\beta$-catenin ${ }^{20,} 21$. Accordingly, a GSEA showed an enrichment in the Cordenonsi YAP Conserved Signature 
consisting in target genes of YAP/TAZ signaling ${ }^{22}$ (Figure 6D, Figure S10A). The partners of YAP/TAZ signaling Yap1, Taz (Wwtr1) and the transcription factors involved in Yap/Tazdependent gene expression: Tead1,2,3,4, were also overexpressed in UNDIFF tumors, (Figure S10B). We thus characterized this pathway in situ in control livers (Figure 7, Figure S11), in DIFF and UNDIFF tumors by IHC (Figure 7). We found a zonal expression of Taz protein in pericentral hepatocytes in control livers. Taz was abundant in DIFF tumors but absent from UNDIFF ones. Tead1 protein was expressed in bile ducts of control livers and at the periphery of DIFF tumors, but was absent from DIFF tumor cells. Conversely, UNDIFF tumors strongly expressed Yap1 and TEAD1, with no expression of Taz. By combining the data showing the overexpression of Yap target genes in UNDIFF tumors with those showing the colocalization of Yap1 and Tead1 in the nucleus of UNDIFF tumor cells, we concluded that a Yap/Tead1 signaling cascade promotes the expression of oncogenic Yap targets in UNDIFF mouse tumors.

\section{Discussion}

Mouse models of $\beta$-catenin dependent tumorigenesis have been widely used. However, while HCC development can be induced by bi-allelic loss-of-function of Apc in mice, it was believed since 2002 that the expression of a dominant stable mutant of $\beta$-catenin is not sufficient to induce tumorigenesis ${ }^{10}$. It was supposed that liver carcinogenesis requires additional oncogenic events to emerge, justifying the development of new models of $\beta$ catenin-activated oncogenesis, in which the gain-of-function of $\beta$-catenin was combined to that of $\mathrm{H}-\mathrm{Ras}^{23}, \mathrm{C}^{2} \mathrm{Met}^{24}, \mathrm{Akt}^{25}$ or $\mathrm{YAP}^{21}$. These models brought significant information concerning the oncogenic cooperations involved with $\beta$-catenin signaling, which speed up tumor emergence, and emphasized the fact that $\beta$-catenin activation is not oncogenic per se in the liver. Moreover, this observation raised interrogations regarding the relevance of mouse models using Apc loss-of-function.

Here we first showed that $\beta$ cat ${ }^{\Delta \mathrm{ex} 3}$ mouse model can actually induce tumorigenesis without engineering additional genetic alterations in mice, suggesting that $\beta$-catenin activation is an initiating event for liver carcinogenesis, even if a latency period of 6 to 9 months is observed before tumor detection. This latency period can be due to the fact that activating $\beta$-catenin signaling does not promote proliferation in single hepatocytes that 
remain quiescent, but changes their metabolic and epigenetic properties ${ }^{13,26,27}$. The oncogenicity of $\beta$-catenin signaling in the liver is thus a longstanding process, which remains to be elucidated ${ }^{28}$. The latency between $\beta$-catenin activation and tumorigenesis can by itself explain why a dominant stable $\beta$-catenin was previously described as not tumorigenic in the liver, due to a 6-month tumor follow-up ${ }^{10}$. On the other hand, as for the Apc model, the number of $\beta c a t^{\Delta \mathrm{ex} 3}$ hepatocytes needs to reach a threshold above which tumor development can be observed during the animal lifetime, while being low enough not to impair liver functions with a subsequent lethality. Interestingly, no mortality, full penetrance and a high reproducibility was observed using CRISPR-mediated exon 3 deletion, showing that this AAVCRISPR approach is the method of choice to generate $\beta$-catenin-activated liver tumors.

Two types of tumors, discriminated by their phenotype (differentiated or undifferentiated), were generated using $A p c^{f s-e x 15}$ or $\beta c t^{\Delta \mathrm{ex} 3}$ models. Transcriptomic and phenotypic analysis clearly show that generated tumors are indistinguishable depending on the model used but are strongly clustering depending on the differentiation status. This first supports the hypothesis that $A p c$ loss-of-function drives hepatocarcinogenesis through the activation of $\beta$-catenin signaling, meaning that the alternative pathways in which Apc is involved, such as chromosomal instability promoted by Apc loss in colorectal cancers, does not prevail in $\mathrm{HCC}$ initiation ${ }^{8}$. Second, our data show two distinct oncogenesis pathways driven by $\beta$-catenin signaling in the liver. Integrated analysis of murine samples with human HCC and hepatoblastomas indicates that mouse differentiated tumors are transcriptionally close to G5G6 human HCCs which are well differentiated and mutated for CTNNB1. Murine undifferentiated tumors cluster with human hepatoblastomas mostly mutated in CTNNB1. Interestingly, the three mesenchymal human hepatoblastoma, two of which being CTNNB1 mutated, are closer to murine undifferentiated tumors. The origin of hepatoblastoma-like tumors in our models is unknown. The development of HCC and hepatoblastoma following expression in fetal liver progenitor cells of a stable mutant of $\beta$-catenin has been already described in mice, raising the possibility that undifferentiated tumors arise from immature/progenitor cells in the liver ${ }^{29}$. This would suggest that in our study, liver progenitors could be targeted by the Cre-lox system or AAV vectors to deliver the CRISPR machinery. However, AAV8 vectors coupled with TBG promoter have been described highly specific in targeting hepatocytes without targeting any other hepatic compartment ${ }^{25}$. Moreover, the 
TTR-CreTam mouse used for CreLox strategy was described to be active both in the fetal and the adult liver, but we could show $\beta$-catenin accumulation only in hepatocytes, after Tamoxifen injection to adult mice ${ }^{13,14}$. In agreement with this, previous studies support the fact that HCC originates from hepatocytes and not from progenitor/biliary compartment ${ }^{30}$. Lastly more and more studies suggest that hepatocytes and cholangiocytes are the main sources of hepatic progenitors in the liver ${ }^{31-33}$, and this suggests that both differentiated and undifferentiated tumor types arise from mature hepatocytes. We hypothesize that different additional oncogenic events elicited by $\beta$-catenin activation in hepatocytes, could dictate the path toward a more or less differentiated cell fate and lead to specific tumor phenotypes.

Our data also strengthen the hypothesis that distinct Yap/Taz signalings could be players of either differentiated HCCs or poorly differentiation liver tumors. Yap and Taz are not identical twins, as recently reviewed in other biological systems ${ }^{34}$. In the liver, one cascade could be dictated by Taz together with $\beta$-catenin: Taz expression is limited to $\beta$-catenin-activated pericentral hepatocytes in control livers, and it is found together with Yap1 in $\beta$-cateninactivated DIFF mouse tumors. This suggests that Taz and $\beta$-catenin could be interactive inducers of pericentral gene expression, both in pericentral hepatocytes, and in DIFF mouse tumors and human G5/G6 $\mathrm{HCCs}^{3}$. On the other hand, Yap1 has been described as overriding the pericentral zonation program and directing periportal hepatocytes to dedifferentiate into atypical ductal cells $s^{35}$. Accordingly, we found here that immunostainings for both Yap1 and its nuclear effector Tead1 are more intense in and around the bile ducts. They are co-expressed in $\beta$-catenin-activated UNDIFF tumors, which exhibit small and basophilic tumor cells with atypical ductal cells features. This strongly suggests a Yap1/Tead1 cascade in UNDIFF tumors, and potentially in Hepatoblastomas.

Lastly, we described the possibility to use CRISPR-mediated gene editing for studying long term tumorigenesis. Some concerns have been raised regarding the persistence of edited hepatocytes due to the immunogenic properties of the SaCas9. While a recent study describes the loss of edited hepatocytes with time in a model of SaCas9 pre-immunized mice, we did not observe such a decrease $\mathrm{e}^{36}$. Another concern regarding in vivo editing using CRISPR is the specificity of the edition. We found an unwanted outcome, as the sequencing of one $\beta c^{\Delta t^{\Delta e x 3}}$ tumor revealed the insertion of $42 \mathrm{bp}$ similar to the AAV ITR. Insertion of AAV have been: (1) described as potential oncogenic events in human $\mathrm{HCC}^{37}, 38$; (2) described as common at 
CRISPR-induced Double-Stranded Breaks ${ }^{39}$. A retrospective study on the use of AAV vector in therapy (not combined with CRISPR) clearly described AAV vectors as safe in clinical settings ${ }^{40}$. Unwanted tumorigenesis due to AAV insertion in mouse experimental settings have not been reported yet. Altogether the easy setting, and time saving aspect of CRISPR mediated in vivo gene editing compared to the development of new Cre-lox models makes it very attractive to study liver carcinogenesis ${ }^{41}$.

\section{Acknowledgements}

We thank Pr Feng Zhang for the gift of AAV8-CRISPR-SaCas9 plasmids, and Dr Violaine Moreau for helpful discussions. We are grateful to the animal facilities of Cochin Institute and of the Centre de Recherche des Cordeliers for mice care, and to the Live Imaging Platform of Cochin Institute for ultrasound follow-up. We also thank Yoan Renoux-Martin for Yap, Taz and Tead1 immunostainings.

\section{References :}

1. Calderaro J, Ziol M, Paradis V, et al. Molecular and histological correlations in liver cancer. J Hepatol 2019;71:616-630.

2. de La Coste $A$, Romagnolo B, Billuart $P$, et al. Somatic mutations of the beta-catenin gene are frequent in mouse and human hepatocellular carcinomas. Proc Natl Acad Sci U S A 1998;95:8847-51.

3. Gougelet A, Torre C, Veber P, et al. T-cell factor 4 and beta-catenin chromatin occupancies pattern zonal liver metabolism in mice. Hepatology 2014;59:2344-57.

4. Rebouissou S, Franconi A, Calderaro J, et al. Genotype-phenotype correlation of CTNNB1 mutations reveals different ss-catenin activity associated with liver tumor progression. Hepatology 2016;64:2047-2061.

5. Schulze K, Imbeaud S, Letouze E, et al. Exome sequencing of hepatocellular carcinomas identifies new mutational signatures and potential therapeutic targets. Nat Genet 2015;47:505-511.

6. Abitbol S, Dahmani R, Coulouarn C, et al. AXIN deficiency in human and mouse hepatocytes induces hepatocellular carcinoma in the absence of beta-catenin activation. J Hepatol 2018;68:1203-1213.

7. Colnot S, Decaens T, Niwa-Kawakita M, et al. Liver-targeted disruption of Apc in mice activates beta-catenin signaling and leads to hepatocellular carcinomas. Proc Natl Acad Sci U S A 2004;101:17216-21.

8. Fodde R, Smits R, Clevers H. APC, signal transduction and genetic instability in colorectal cancer. Nat Rev Cancer 2001;1:55-67.

9. Armengol $C$, Cairo $S$, Fabre $M$, et al. Wnt signaling and hepatocarcinogenesis: the hepatoblastoma model. Int J Biochem Cell Biol 2011;43:265-70. 
bioRxiv preprint doi: https://doi.org/10.1101/2021.07.04.450836; this version posted July 5, 2021. The copyright holder for this preprint (which was not certified by peer review) is the author/funder. All rights reserved. No reuse allowed without permission.

Loesch et al., J Hepatol

10. Harada N, Miyoshi $\mathrm{H}$, Murai N, et al. Lack of tumorigenesis in the mouse liver after adenovirus-mediated expression of a dominant stable mutant of beta-catenin. Cancer Res 2002;62:1971-7.

11. Benhamouche $S$, Decaens T, Godard C, et al. Apc tumor suppressor gene is the "zonationkeeper" of mouse liver. Dev Cell 2006;10:759-70.

12. Gougelet A, Sartor C, Bachelot L, et al. Antitumour activity of an inhibitor of miR-34a in liver cancer with beta-catenin-mutations. Gut 2016;65:1024-34.

13. Gougelet A, Sartor C, Senni N, et al. Hepatocellular Carcinomas With Mutational Activation of Beta-Catenin Require Choline and Can Be Detected by Positron Emission Tomography. Gastroenterology 2019;157:807-822.

14. Ran FA, Cong L, Yan WX, et al. In vivo genome editing using Staphylococcus aureus Cas9. Nature 2015;520:186-91.

15. Tannour-Louet $\mathrm{M}$, Porteu $\mathrm{A}$, Vaulont $\mathrm{S}$, et al. A tamoxifen-inducible chimeric Cre recombinase specifically effective in the fetal and adult mouse liver. Hepatology 2002;35:1072-81.

16. Hirsch TZ, Pilet J, Morcrette G, et al. Integrated genomic analysis identifies driver genes and cisplatin-resistant progenitor phenotype in pediatric liver cancer. Cancer Discov 2021.

17. Boyault S, Rickman DS, de Reynies A, et al. Transcriptome classification of HCC is related to gene alterations and to new therapeutic targets. Hepatology 2007;45:42-52.

18. Calderaro J, Couchy G, Imbeaud S, et al. Histological subtypes of hepatocellular carcinoma are related to gene mutations and molecular tumour classification. J Hepatol 2017;67:727738.

19. Decaens T, Godard C, de Reynies A, et al. Stabilization of beta-catenin affects mouse embryonic liver growth and hepatoblast fate. Hepatology 2008;47:247-58.

20. Sylvester KG, Colnot S. Hippo/YAP, beta-catenin, and the cancer cell: a "menage a trois" in hepatoblastoma. Gastroenterology 2014;147:562-5.

21. Tao J, Calvisi DF, Ranganathan S, et al. Activation of beta Catenin and Yap1 in Human Hepatoblastoma and Induction of Hepatocarcinogenesis in Mice. Gastroenterology 2014.

22. Cordenonsi M, Zanconato F, Azzolin L, et al. The Hippo transducer TAZ confers cancer stem cell-related traits on breast cancer cells. Cell 2011;147:759-72.

23. Harada $\mathrm{N}$, Oshima $\mathrm{H}$, Katoh $\mathrm{M}$, et al. Hepatocarcinogenesis in mice with beta-catenin and Haras gene mutations. Cancer Res 2004;64:48-54.

24. Tward AD, Jones KD, Yant $S$, et al. Distinct pathways of genomic progression to benign and malignant tumors of the liver. Proc Natl Acad Sci U S A 2007;104:14771-6.

25. Stauffer JK, Scarzello AJ, Andersen JB, et al. Coactivation of AKT and beta-catenin in mice rapidly induces formation of lipogenic liver tumors. Cancer Res 2011;71:2718-27.

26. Senni N, Savall M, Cabrerizo Granados D, et al. beta-catenin-activated hepatocellular carcinomas are addicted to fatty acids. Gut 2019;68:322-334.

27. Torre $C$, Benhamouche $S$, Mitchell $C$, et al. The transforming growth factor-alpha and cyclin D1 genes are direct targets of beta-catenin signaling in hepatocyte proliferation. J Hepatol 2011;55:86-95.

28. Gougelet A, Colnot S. A Complex Interplay between Wnt/beta-Catenin Signalling and the Cell Cycle in the Adult Liver. Int J Hepatol 2012;2012:816125.

29. Mokkapati S, Niopek K, Huang L, et al. beta-catenin activation in a novel liver progenitor cell type is sufficient to cause hepatocellular carcinoma and hepatoblastoma. Cancer Res 2014;74:4515-25.

30. Mu X, Espanol-Suner R, Mederacke I, et al. Hepatocellular carcinoma originates from hepatocytes and not from the progenitor/biliary compartment. J Clin Invest 2015;125:3891903.

31. Schaub JR, Malato Y, Gormond C, et al. Evidence against a stem cell origin of new hepatocytes in a common mouse model of chronic liver injury. Cell Rep 2014;8:933-9. 
bioRxiv preprint doi: https://doi.org/10.1101/2021.07.04.450836; this version posted July 5, 2021. The copyright holder for this preprint (which was not certified by peer review) is the author/funder. All rights reserved. No reuse allowed without permission.

Loesch et al., J Hepatol

32. Tarlow BD, Pelz C, Naugler WE, et al. Bipotential adult liver progenitors are derived from chronically injured mature hepatocytes. Cell Stem Cell 2014;15:605-18.

33. Yanger $\mathrm{K}$, Knigin $\mathrm{D}$, Zong $\mathrm{Y}$, et al. Adult hepatocytes are generated by self-duplication rather than stem cell differentiation. Cell Stem Cell 2014;15:340-349.

34. Reggiani F, Gobbi G, Ciarrocchi A, et al. YAP and TAZ Are Not Identical Twins. Trends Biochem Sci 2021;46:154-168.

35. Fitamant J, Kottakis F, Benhamouche S, et al. YAP Inhibition Restores Hepatocyte Differentiation in Advanced HCC, Leading to Tumor Regression. Cell Rep 2015;10:1692-1707.

36. Li A, Tanner MR, Lee CM, et al. AAV-CRISPR Gene Editing Is Negated by Pre-existing Immunity to Cas9. Mol Ther 2020;28:1432-1441.

37. La Bella T, Imbeaud S, Peneau C, et al. Adeno-associated virus in the liver: natural history and consequences in tumour development. Gut 2020;69:737-747.

38. Nault JC, Datta S, Imbeaud S, et al. Recurrent AAV2-related insertional mutagenesis in human hepatocellular carcinomas. Nat Genet 2015;47:1187-93.

39. Hanlon KS, Kleinstiver BP, Garcia SP, et al. High levels of AAV vector integration into CRISPRinduced DNA breaks. Nat Commun 2019;10:4439.

40. Naso MF, Tomkowicz B, Perry WL, 3rd, et al. Adeno-Associated Virus (AAV) as a Vector for Gene Therapy. BioDrugs 2017;31:317-334.

41. Loesch R, Desbois-Mouthon C, Colnot S. Potentials of CRISPR in liver research and therapy. Clin Res Hepatol Gastroenterol 2018. 
A

\begin{tabular}{|l|c|c|c|}
\hline Tamoxifen & 1mg IP & $\mathbf{0 , 5 m g ~ I P ~}$ & Diet 400mg/2days \\
\hline Mice (n) & 26 & 12 & 20 \\
\hline Surviving mice (n, [\%]) & $6[23 \%]$ & $7[58 \%]$ & $18[90 \%]$ \\
\hline Sacrifice (post Tam) & 12 months & 12 months & 10 months \\
\hline Mice with Tumors (n) & 4 & 4 & 11 \\
\hline Penetrance & $67 \%$ & $57 \%$ & $61 \%$ \\
\hline
\end{tabular}

B

$\beta$-Catenin
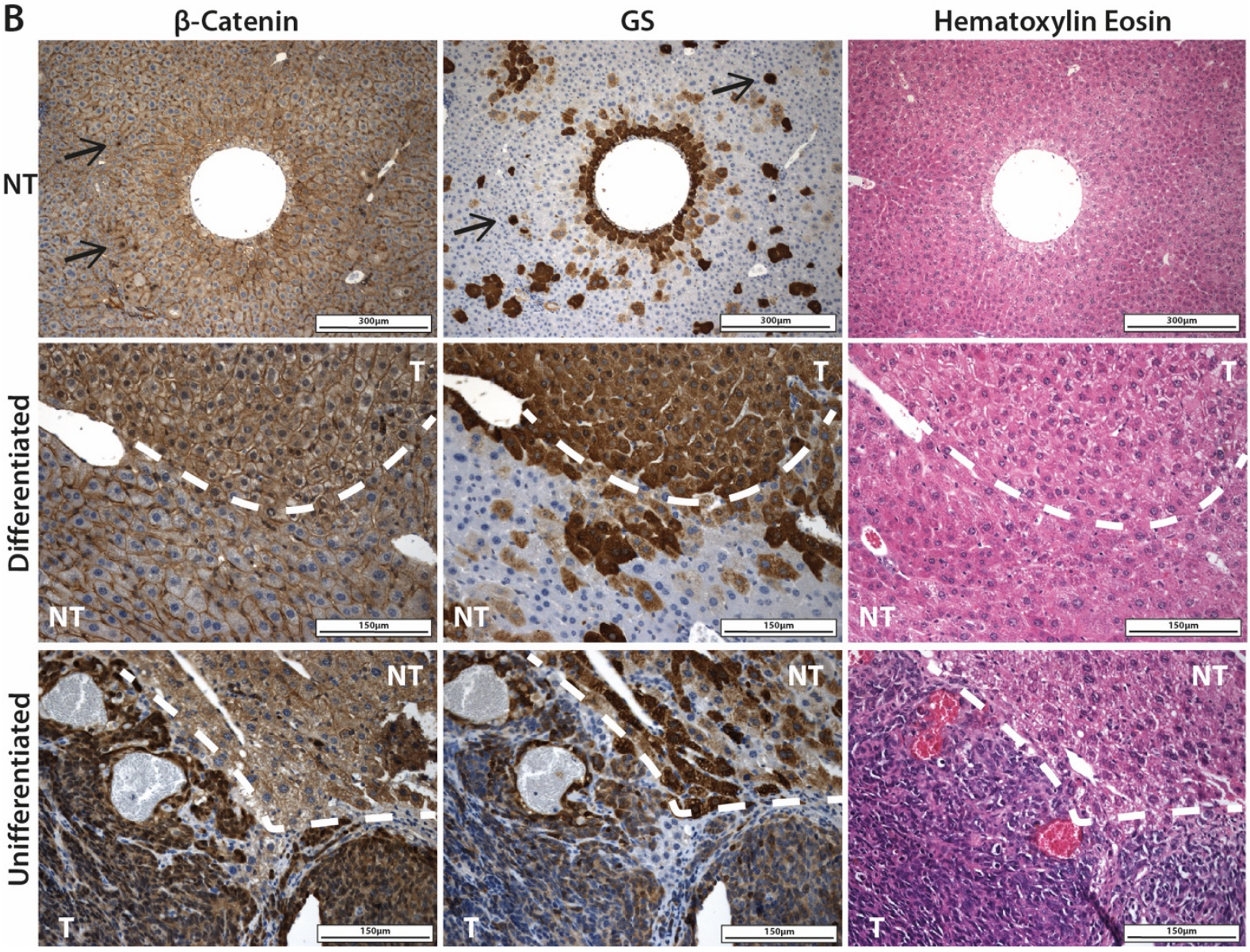

Figure 1: Emergence of differentiated and undifferentiated tumors after Tamoxifen-induced hepatic-specific loss of $\boldsymbol{\beta}$-catenin-exon 3. A: Mice used in the study; B: Representative hematoxyline-Eosine stainings and IHC for $\beta$-catenin, glutamine synthetase (GS), on non-tumoral tissue (NT) and tumoral tissue. Black arrows: $\beta$-catenin activated hepatocytes. 
A
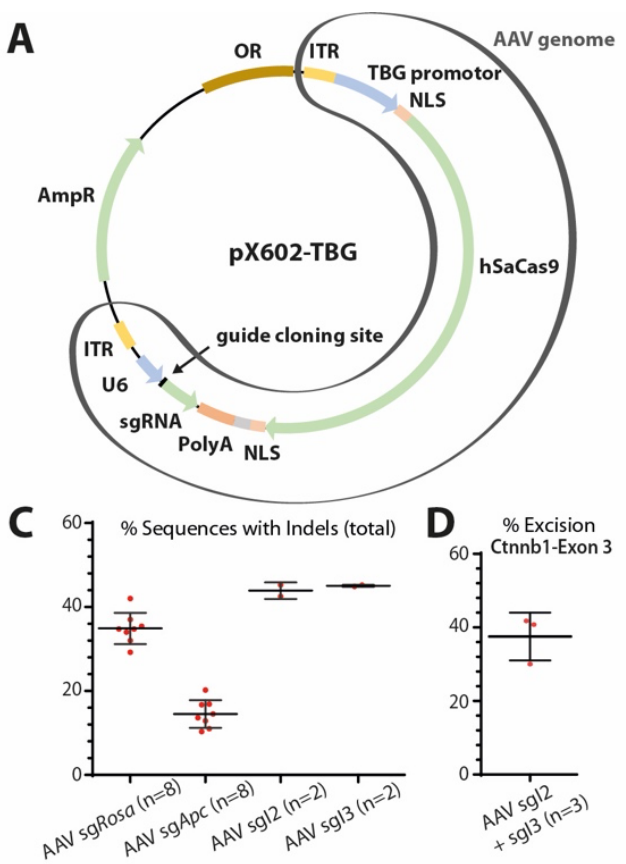

B
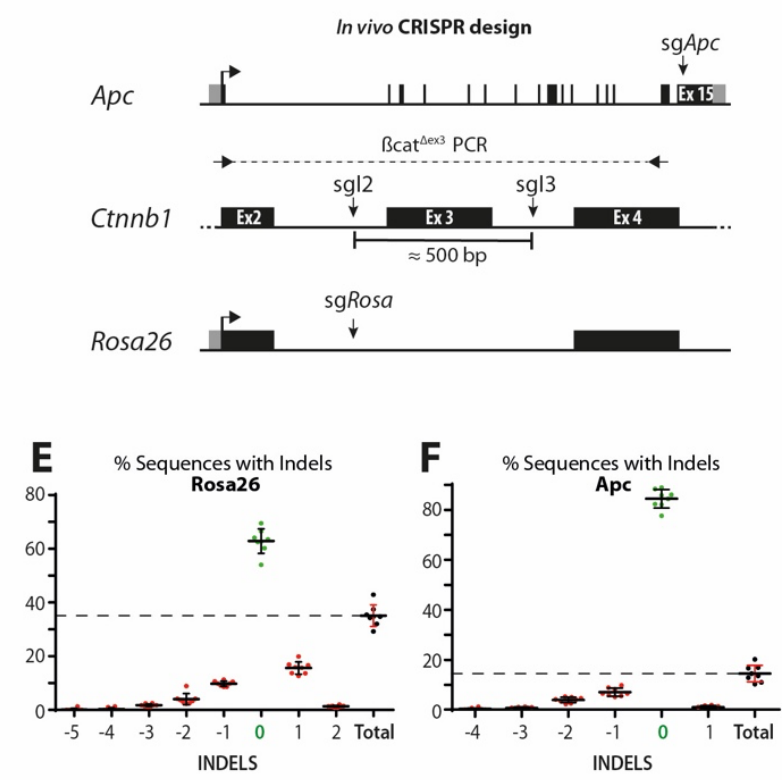

G

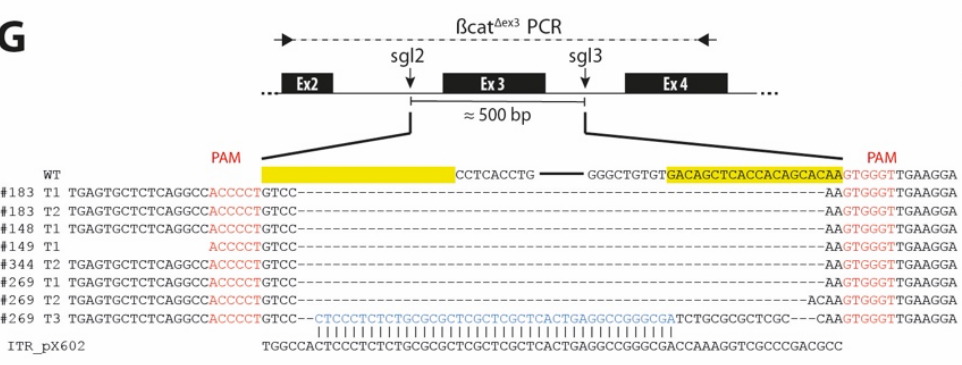

H Percent of tumor-free mice after AAV-CRISPR

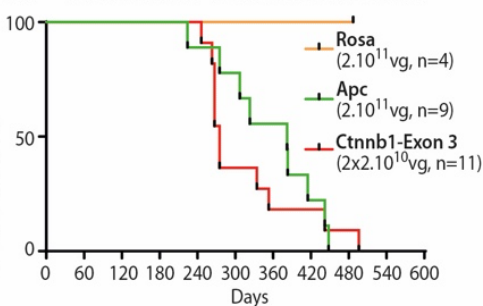

I

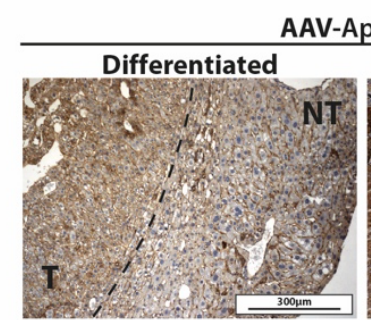

Indel spectrum in tumor

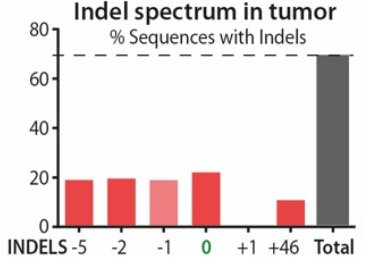

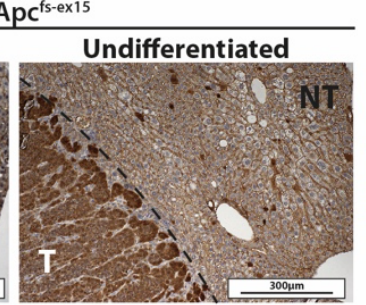

Indel spectrum in tumor

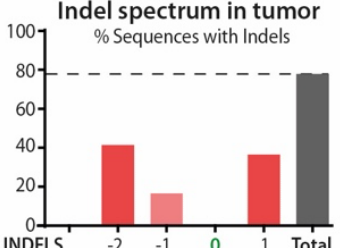

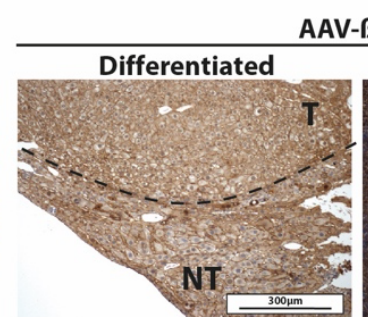

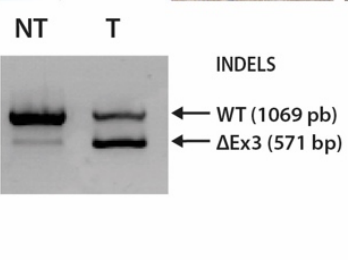

AV-ßcat ${ }^{\Delta e x 3}$

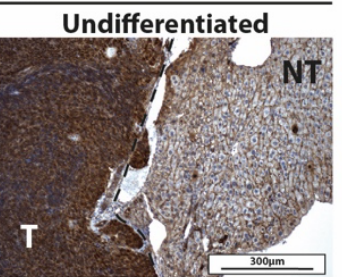

NT T

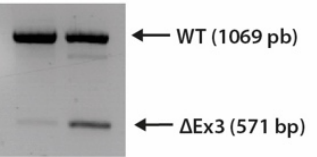

Figure 2: Tumor formation after AAV-CRISPR/Cas9 mediated loss-of-function of Apc or gainof-function of $\boldsymbol{\beta}$-catenin. A: pX602-TBG plasmid for the production of hepatotropic AAV8 vectors encoding the SaCas9 driven by a hepatospecific TBG promoter and a guide RNA. Small guides targeting Apc, Ctnnb1-Intron 2 (sgl2), Ctnnb1-Intron 3 (sgl3) or Rosa26 locus (sgRosa) were each cloned into the plasmid. B: In vivo gene editing design. C,D: Total liver gene editing efficiency after AAV injection $\left(2.10^{11} \mathrm{vg}\right.$ total), assessed: (C) after 2 months via Tracking of indels by decomposition (TIDE); (D) after 1 month, via excision of Ctnnb1-Exon 3 (sgl2 + sgl3). E-F: Indel spectrum frequency on total liver 2 months post targeting of Rosa26 or Apc. 0 indel corresponds to the WT sequence ; -1 to -5 corresponds to a deletion of 1 to 5 nucleotides; +1 to +46 to an insertion of 1 to 46 nucleotides. G: Ctnnb1-Exon 3 editing in 8 tumors. H: kinetics of tumor detection by ultrasound. I: $\beta$-catenin staining of tumors induced via CRISPR-Apc $c^{f-e x 15}$ or CRISPR- $\beta$ cat $^{\Delta \mathrm{ex} 3}$ (up) along with tumor editing (down) assessed via TIDE for $A p c^{f s-e x 15}$ and PCR for $\beta$ cat $^{\Delta \mathrm{ex} 3}$. 
A

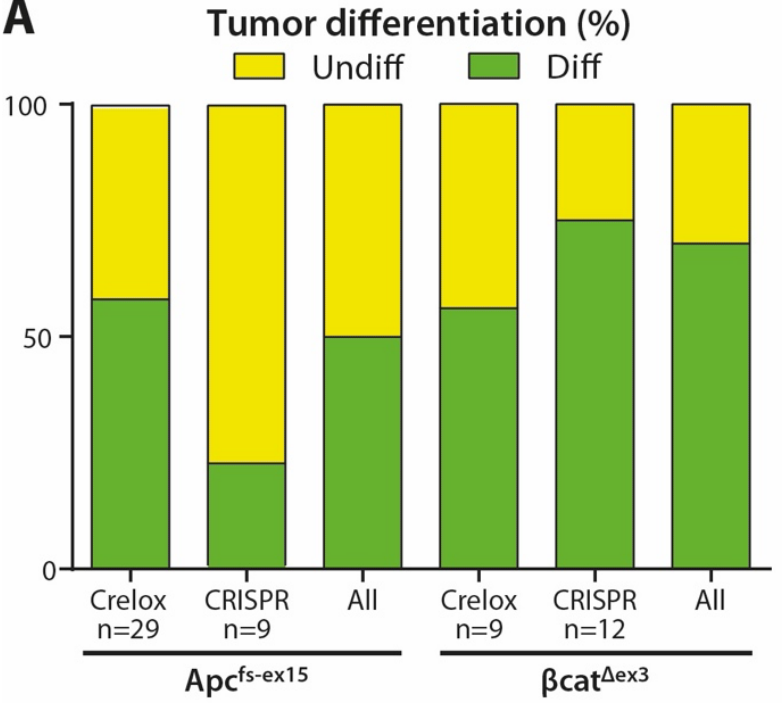

B Tumor size (diameter in mm)

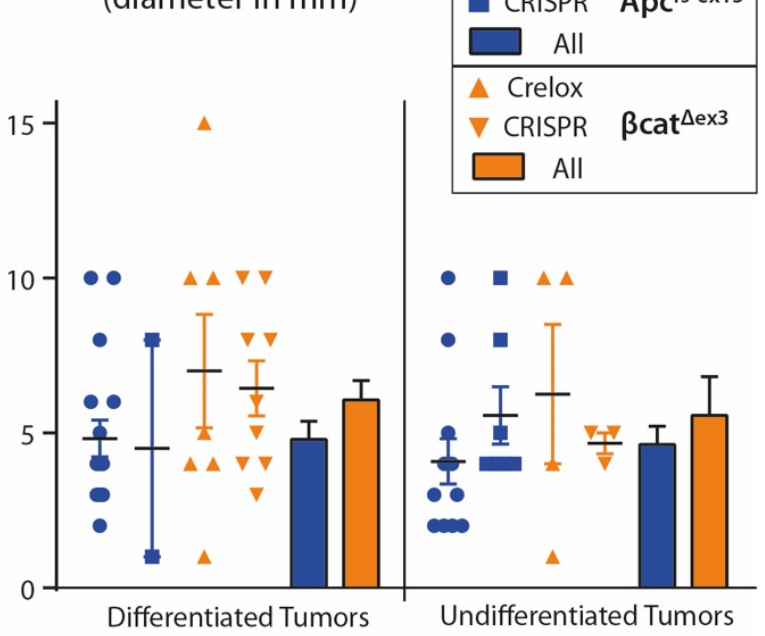

C $\mathrm{Apc}^{\mathrm{fs}-\mathrm{ex} 15}$
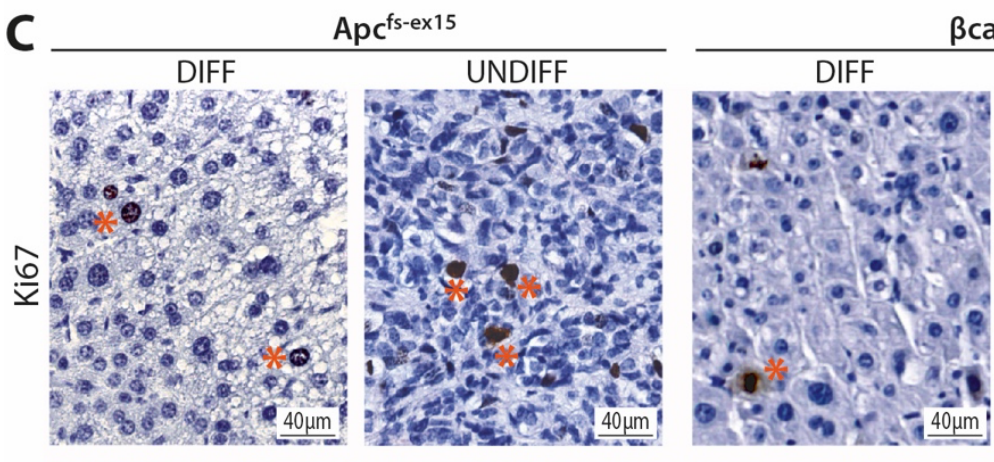
$\beta_{\text {cat }}{ }^{\Delta e x 3}$
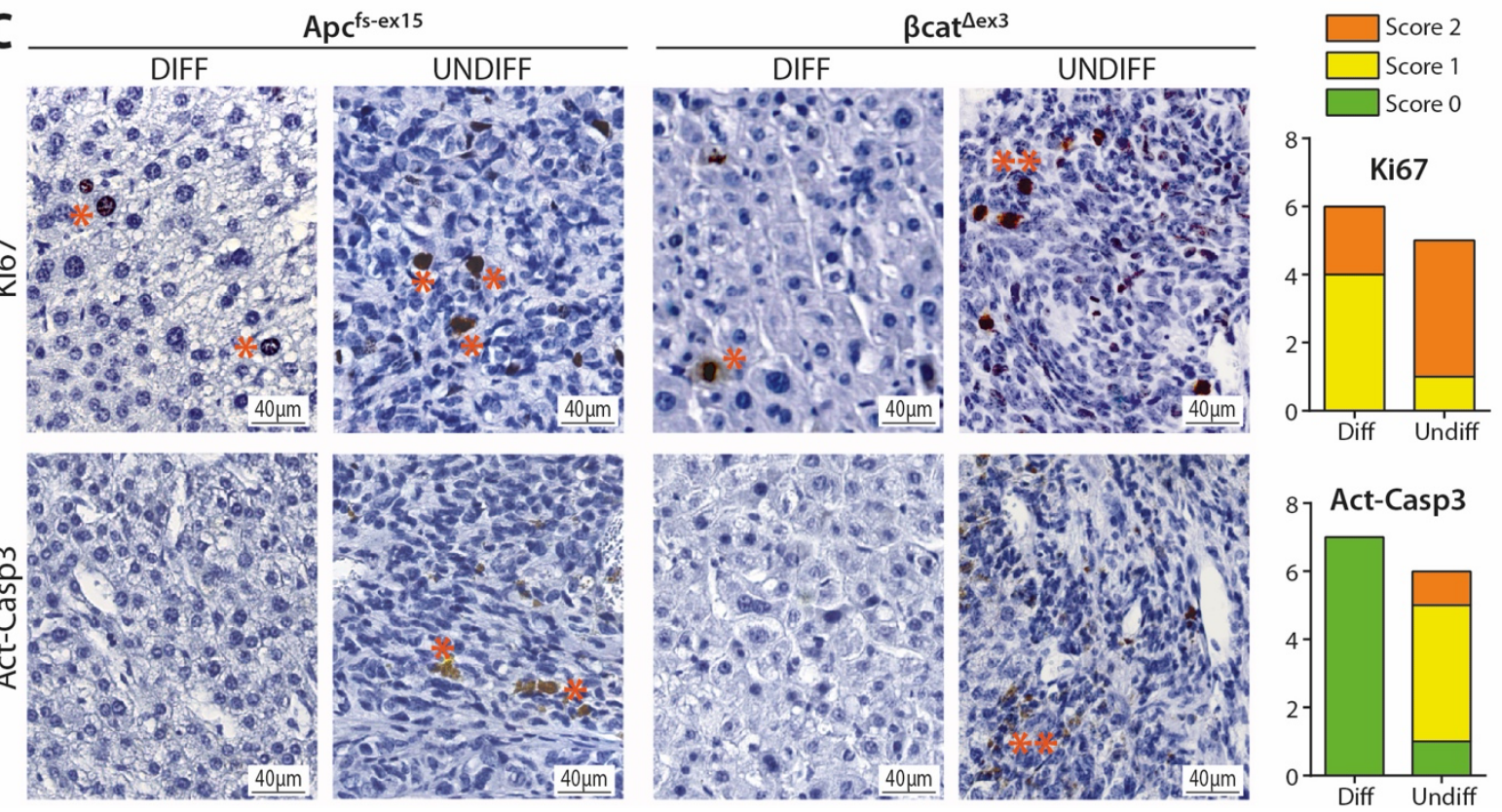

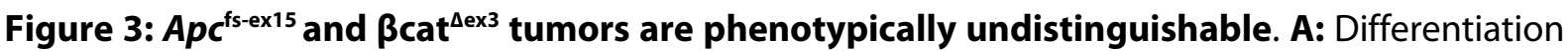
status in tumors from Crelox- and AAV-CRISPR-engineered $A p c^{f 5-e x 15}$ and $\beta c a t^{\Delta \times x 3}$ models. A chisquare test of independence shows no significant association between the genotype and differentiation status, $\chi^{2}(1, N=60)=3.34, p>0,05$. B: Tumor size is not statistically different between the models (One-way ANOVA). C: Proliferation (Ki67) and Apoptosis (ActivatedCaspase 3 ) in tumors. Ki67 immunostaining reveals no statistically significant changes between differentiated and undifferentiated tumors, whereas activated Caspase 3 staining shows that a slight apoptosis can be detected only in undifferentiated tumors. Ki67 and Casp3 IHC were done on 7 diff. tumors from $5 \mathrm{Apc}^{\text {fs-ex15 }}$ (4 Crelox and 1 CRISPR) and $2 \beta_{\text {cat }^{\Delta e x 3}}$ (Crelox)

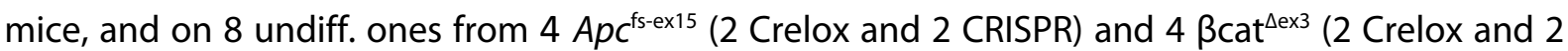
CRISPR) mice. No difference of staining was seen between tumors of $A p c^{f s-e x 15}$ versus $\beta c a t^{\Delta e \times 3}$ genotypes. Scores for IHC were established as follows: Score $0=$ no staining, score $1=$ single tumor cells immunostained $(*)$, score $2=$ clusters of immunostained tumor cells $\left({ }^{* *}\right)$. 
A PC1 vs PC2

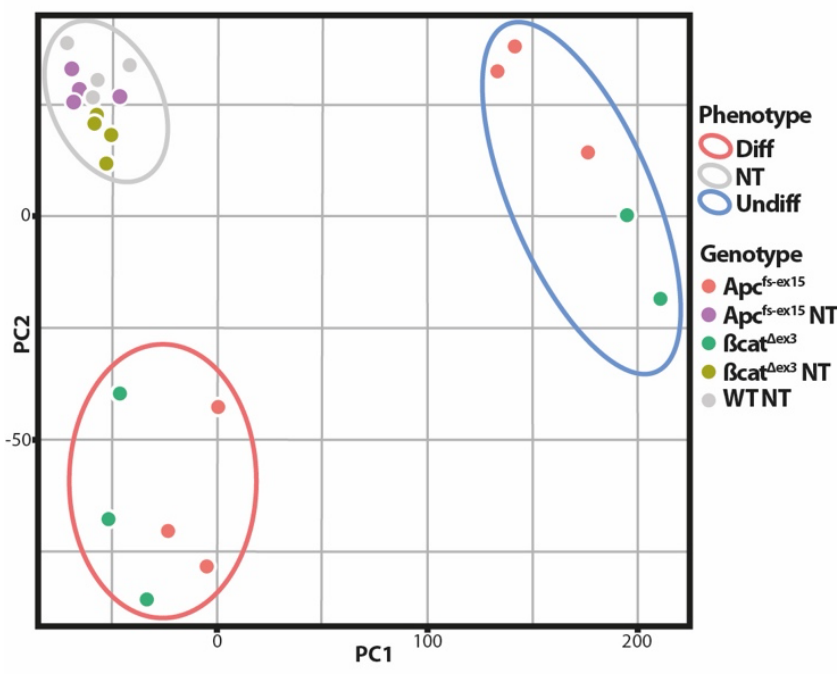

C

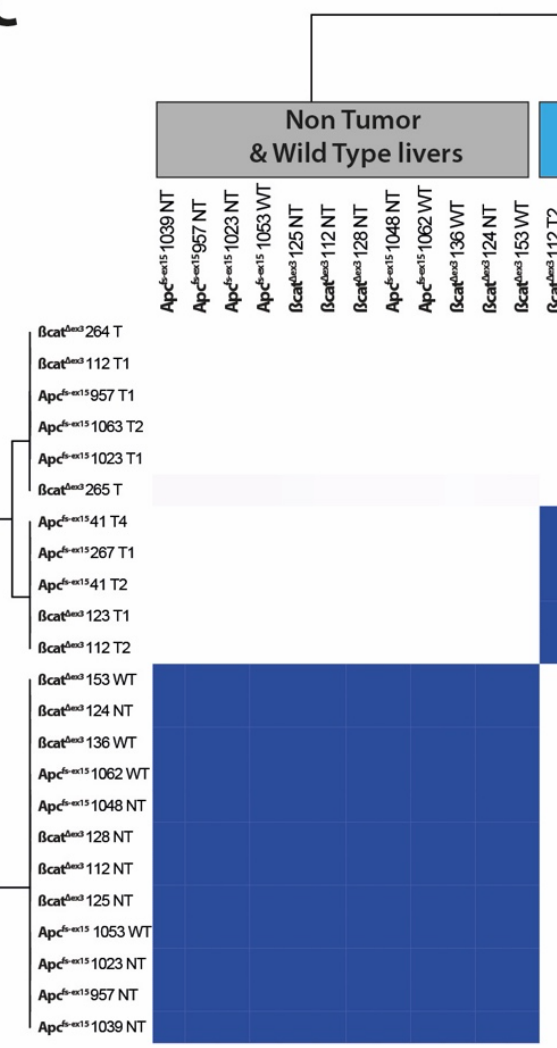

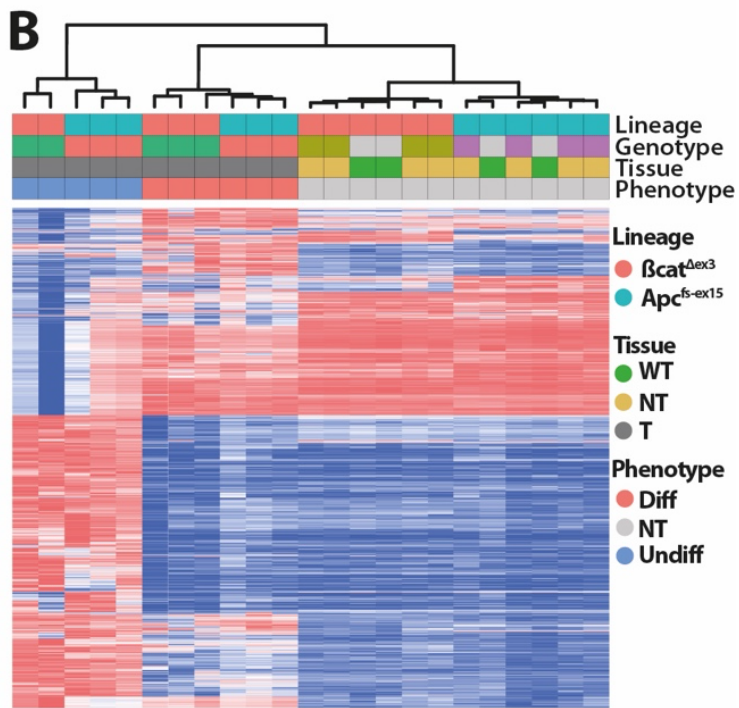

D
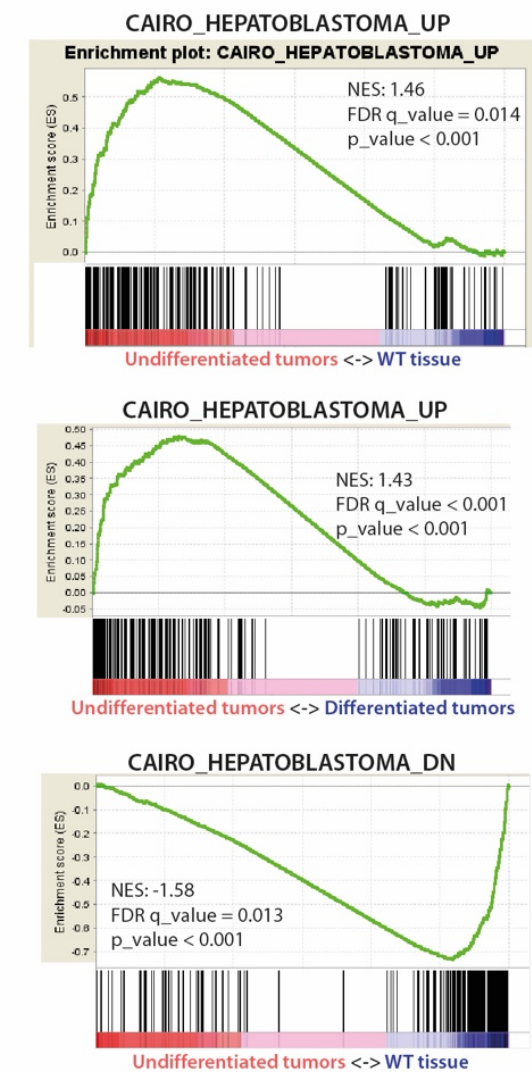

Figure $4: A p c^{f s-e x 15}$ and $\beta$ cat $^{\Delta e x 3}$ tumors clusterize together, depending on their differentiation status. RNAseq data from non-tumoral (NT) wild type (WT) and tumoral tissues from $\mathrm{Apc}^{\mathrm{fs}_{\mathrm{s}} \mathrm{ex} 15}$ and $\beta \mathrm{cat}^{\mathrm{\Delta ex3}}$ models using: A: Principal component analysis; B: unsupervised hierarchical clustering and C: consensus clustering (2000 genes). D: GSEA analysis showing an enrichment of a hepatoblastoma signature in mouse undifferentiated tumors compared to WT tissue or differentiated tumors. 
bioRxiv preprint doi: https://doi.org/10.1101/2021.07.04 450836; this version posted July 5, 2021. The copyright holder for this preprint (which was not certified by peer review) is the author/funder. All rights reserved. No reuse allowed without permission.

Loesch et al.

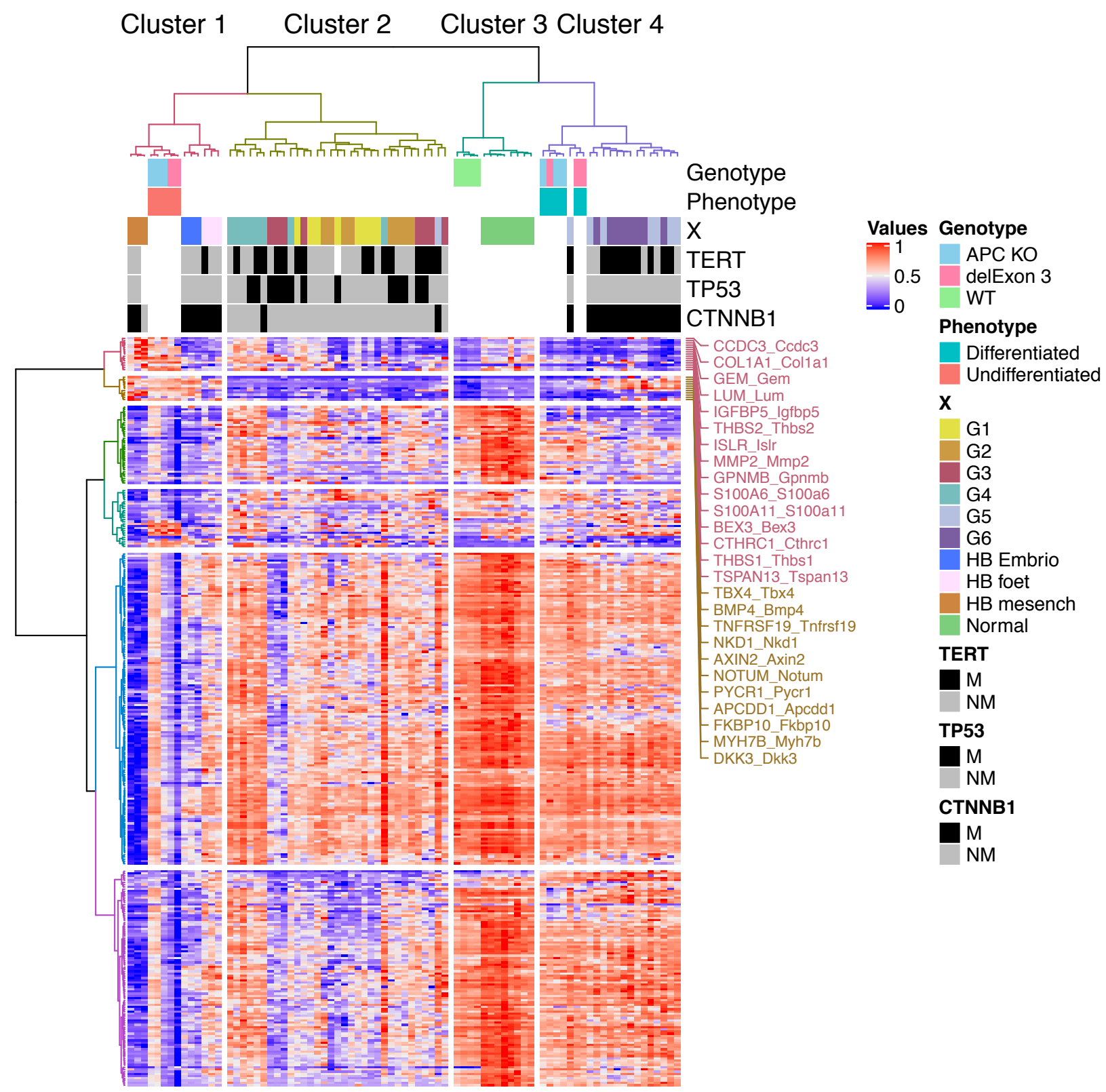

Figure 5: Hierarchical clustering of the integrated human/mouse analysis. The 60 samples are separated into 4 clusters. Cluster 1: Human hepatoblastoma (HB) samples and undifferentiated mouse tumors. Clusters 2: G1 to G4 HCCs. Cluster 3: Human and mouse non-tumoral tissues. Cluster 4: G5 and G6 HCC and differentiated mouse tumors. M, Mutated; NM; non-mutated. 
A
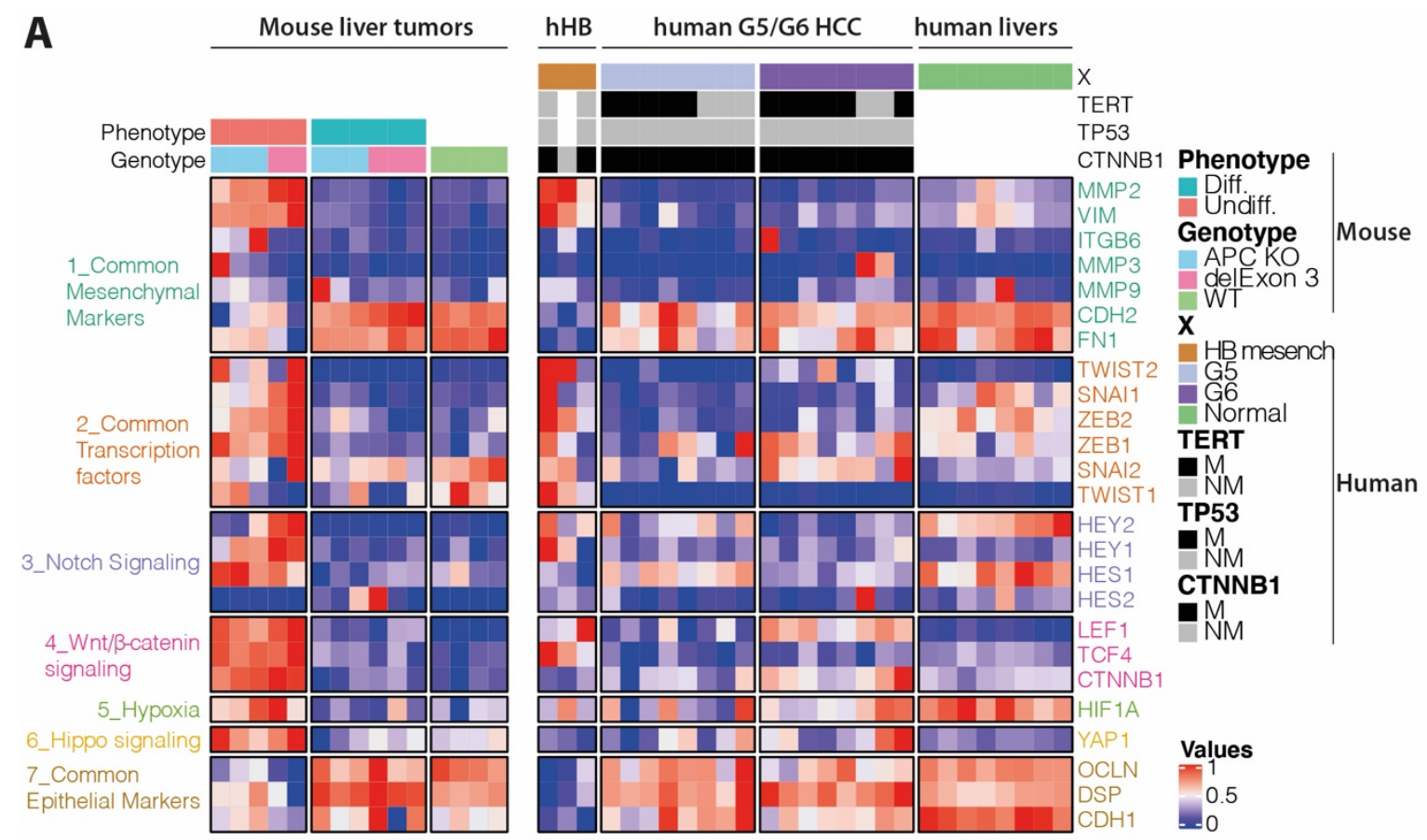

APCKO

$\mathrm{X}$

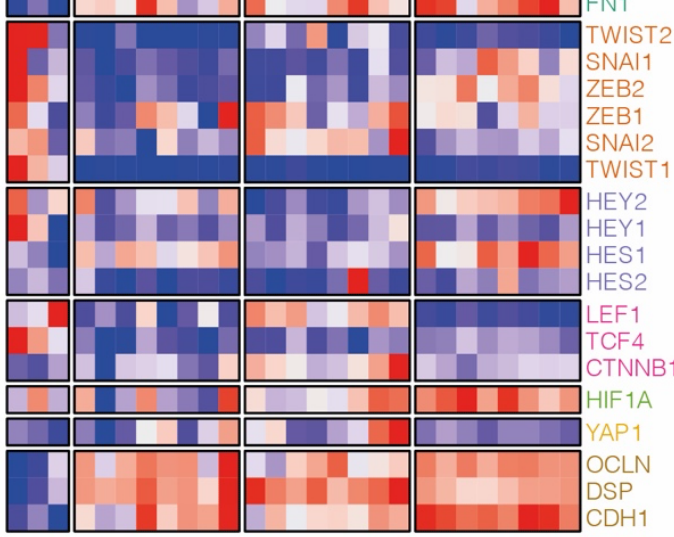

HB mesench

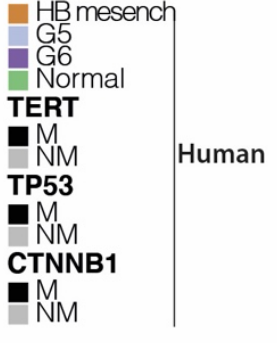

B

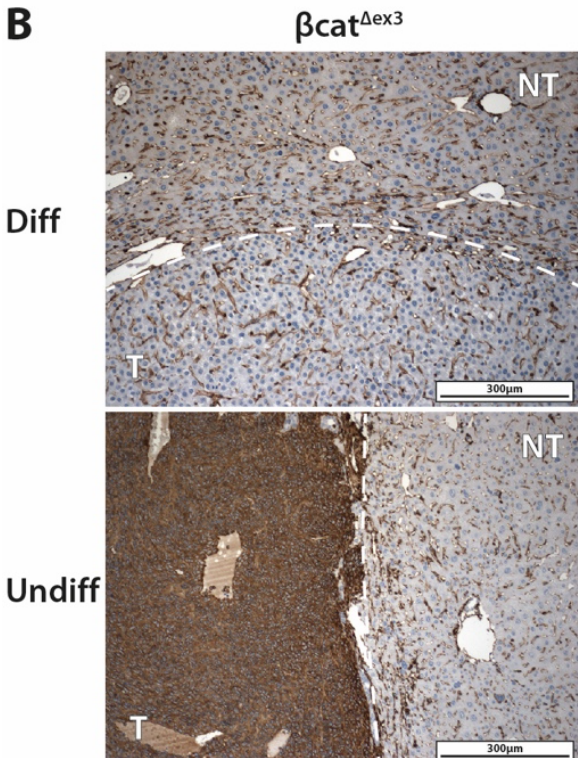

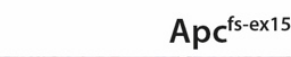

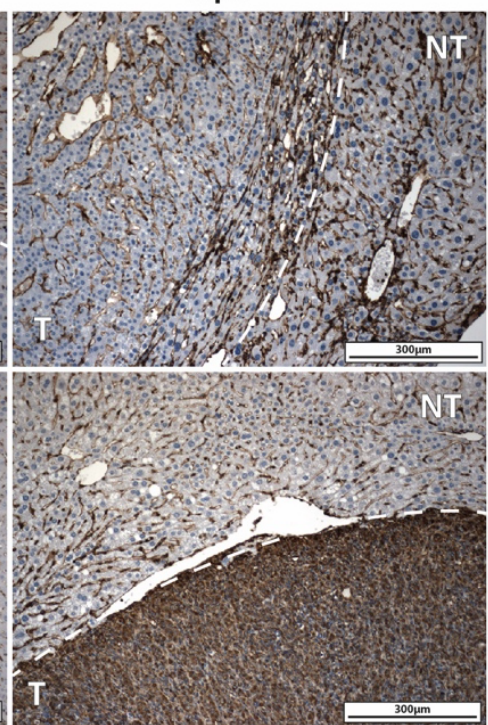

C

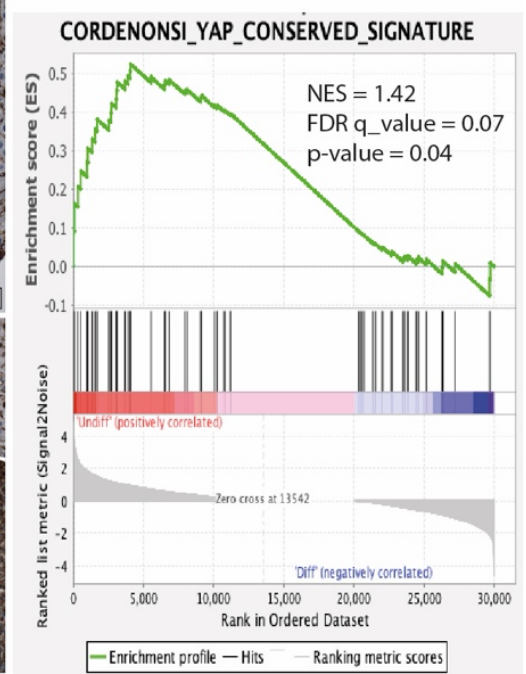

Figure 6: Mesenchymal and YAP signature in undifferentiated $\beta$-catenin-activated mouse

HCCs. A. Expression profile of common EMT related markers in mouse and human samples (Mesenchymal HB, G5-G6 HCCs). B. Vimentin IHC performed on DIFF and UNDIFF mouse tumors from $A p c^{f s-e x 15}$ and $\beta c a t^{\Delta \mathrm{ex} 3}$ models. C. GSEA showing an enrichment in YAP conserved signature in UNDIFF compared to DIFF mouse tumors. 

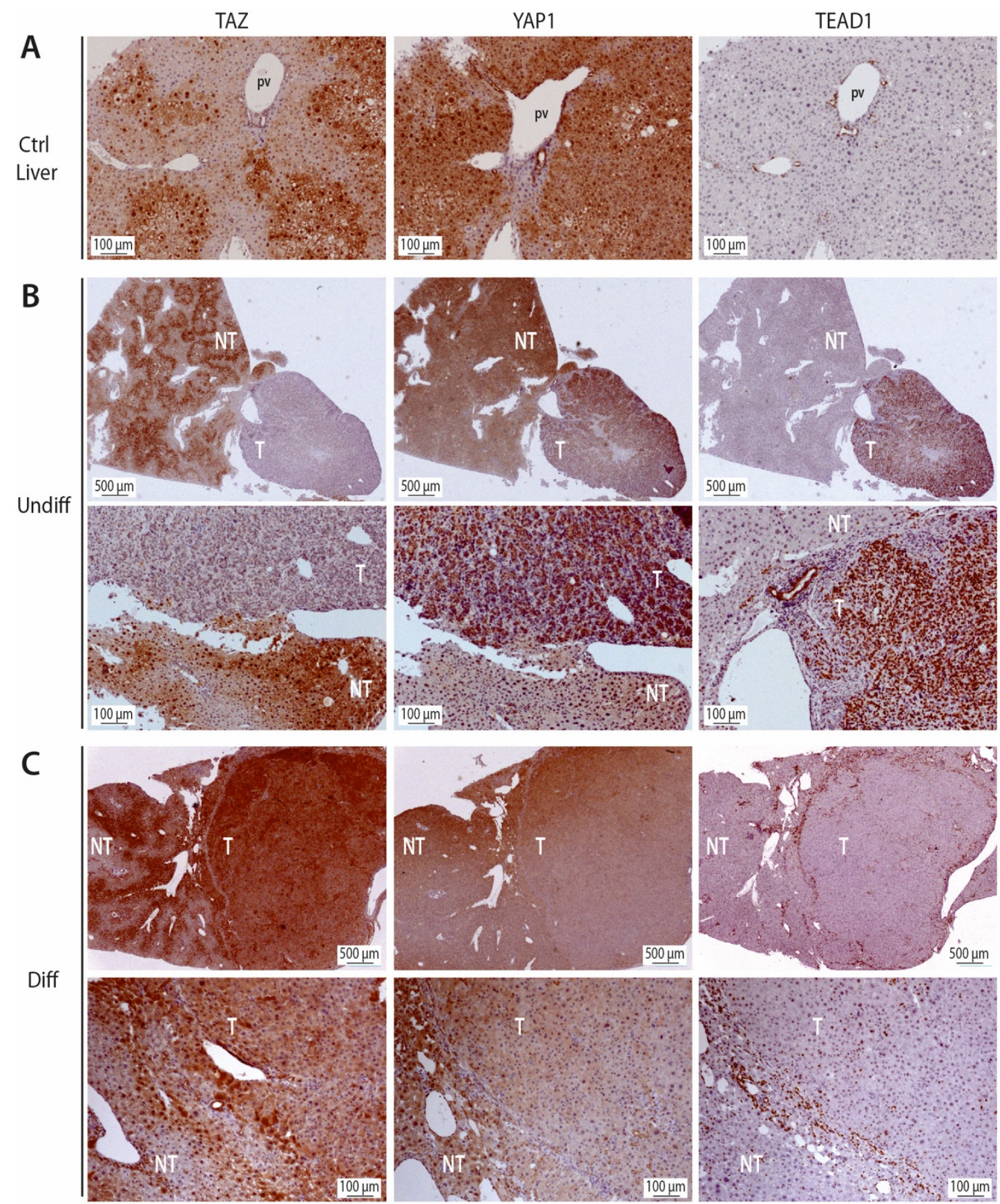

D
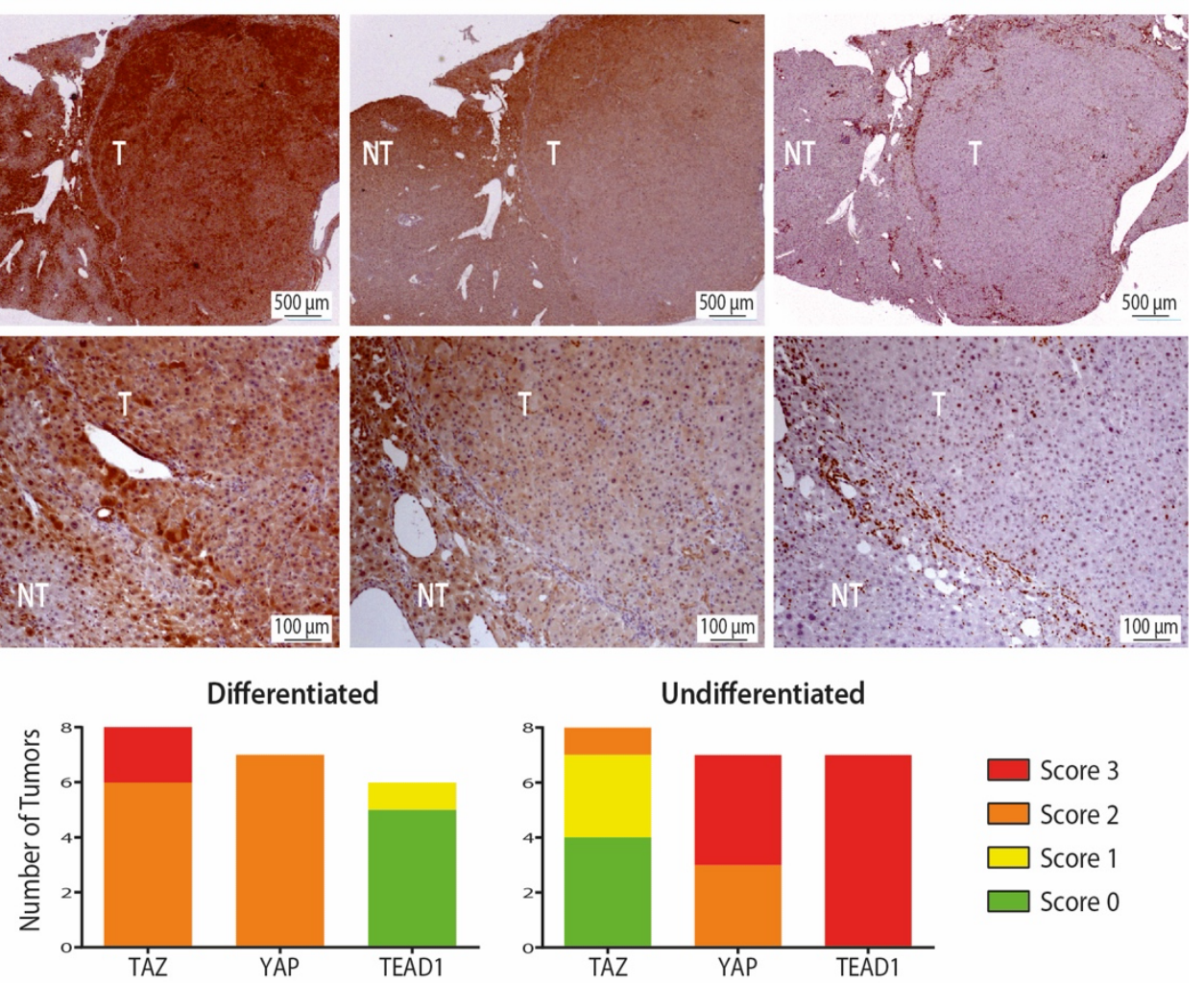

Figure 7: YAP/TAZ pathway is distinctly activated in undifferentiated versus differentiated mouse HCCs. Immunostainings for Taz, Yap1 and Tead1 in representative sections of : A. control liver, B. UNDIFF mouse tumor, C. DIFF mouse tumor. $\mathrm{Pv}=$ portal vein; T=Tumor; NT= Non Tumoral adjacent liver. D. Immunoscorings. Score $0=$ no staining in $T$, score $1=$ staining in $T<N T$, score $2=$ staining in $\mathrm{T}=\mathrm{NT}$, score $3=$ nuclear staining in T $>$ NT. DIFF tumors were from $5 \mathrm{Apc}^{\mathrm{fs}-\mathrm{ex} 15}$ (3 $\mathrm{Crelox}$ and 2 CRISPR) and $2 \beta_{c^{4} t^{\Delta e x 3}}$ ( 1 CRISPR and 2 Crelox) mice. UNDIFF ones came from $5 \mathrm{Apc}^{\text {fs-ex } 15}$ ( 2 Crelox and 3 CRISPR) and $3 \beta$ cat $^{\text {tex3 }}$ ( 2 Crelox and 1 CRISPR) mice. 